\title{
Análise cladística e biogeografia de Ochlerini (Heteroptera, Pentatomidae, Discocephalinae) ${ }^{1}$
}

\author{
Luiz A. Campos ${ }^{2} \&$ Jocélia Grazia ${ }^{3}$
}

1. Contribuição $\mathrm{n}^{0} 479$ do Departamento de Zoologia da UFRGS.

2. Programa de Pós-Graduação em Ciências Ambientais, Universidade do Extremo Sul Catarinense, Av. Universitária 1105, 88806-000 Criciúma, Santa Catarina, Brasil. (lac@unesc.net)

3. Departamento de Zoologia, Universidade Federal do Rio Grande do Sul, Av. Bento Gonçalves 9.500, sl. 223, 91501-970 Porto Alegre, Rio Grande do Sul, Brasil. Bolsista do CNPq. (jocelia@ufrgs.br)

\begin{abstract}
Cladistic analysis and biogeography of Ochlerini (Heteroptera, Pentatomidae, Discocephalinae). A cladistic analysis using 63 characters and 30 genera of the Neotropical tribe Ochlerini Rolston was performed to test their monophyletic condition and to establish a relationship hypothesis. Janeirona Distant, 1911 (Pentatomini) and the tribes Discocephalini and Halyini were included in the ingroup to test their relationship with Ochlerini; Marghita Ruckes, 1964 and Stictochilus Bergroth, 1918 (Pentatomini) were used as outgroups. The obtained strict consensus cladograms indicate that Ochlerini and Discocephalinae are monophyletic groups, but Pentatominae, Halyini+Ochlerini, Pentatomini+Ochlerini, Ochlerus Spinola, 1837, Stalius Rolston, 1992 and Alitocoris Sailer, 1950 are merophyletic groups. Discocephalini and Ochlerini share three synapomorphies: first rostral segment long, attaining prosternum; metasternum with a mesial, longitudinal carina, and dorsal surface of basal third of male proctiger membranous. Ochlerini is supported by one synapomorphy, the flattened dorsal surface of third tarsal segment of hind legs, in females. Biogeographical analysis based on consensus cladograms shows congruent patterns with several vicariant events proposed for the Neotropical region.
\end{abstract}

KEYWORDS. Cladistic analysis, Neotropical region, biogeography, Discocephalinae, Ochlerini.

RESUMO. Neste trabalho foi realizada uma análise cladística, utilizando 63 caracteres, dos 30 gêneros da tribo Neotropical Ochlerini Rolston, no intuito de testar seu monofiletismo e estabelecer uma hipótese de parentesco. Janeirona Distant, 1911 (Pentatomini) e as tribos Discocephalini e Halyini foram incluídas no grupo-interno para testar seu relacionamento com Ochlerini; Marghita Ruckes, 1964 e Stictochilus Bergroth, 1918 (Pentatomini) foram utilizados como grupos-externos. Os cladogramas de consenso estrito obtidos indicam que Ochlerini e Discocephalinae são grupos monofiléticos, mas Pentatominae, Halyini+Ochlerini, Pentatomini+Ochlerini, Ochlerus Spinola, 1837, Stalius Rolston, 1992 e Alitocoris Sailer, 1950 são grupos merofiléticos. Discocephalini e Ochlerini compartilham três sinapomorfias: primeiro segmento do rostro longo, alcançando o prosterno; metasterno carenado longitudinalmente; e superfície dorsal do segmento $\mathrm{X}$ do macho membranosa no terço basal. Ochlerini é sustentada por uma sinapomorfia, a superfície dorsal do terceiro artículo metatarsal das fêmeas aplainada. Uma análise biogeográfica a partir dos cladogramas de consenso mostrou padrões congruentes com eventos vicariantes propostos para a região Neotropical.

PALAVRAS-CHAVE. Análise cladística, região Neotropical, biogeografia, Discocephalinae, Ochlerini.

A família Pentatomidae é, em número de espécies, uma das maiores de Heteroptera, sendo cosmopolita e com maior diversidade nos trópicos. Pentatomidae conta com aproximadamente 760 gêneros e 4.100 espécies (Schuh \& Slater, 1995). Deste total, a subfamília neotropical Discocephalinae apresenta 47 gêneros em Discocephalini e 28 em Ochlerini e, apesar de carecer de estudos cladísticos, tem limites bem estabelecidos e não sofreu alterações notáveis desde sua proposição por FIEBER (1860), até a inclusão de Ochlerini por Rolston (1981), subdividindo-a em duas tribos.

Ochlerini foi proposta para incluir 23 dos 26 gêneros neotropicais removidos de Halyini (Pentatomidae, Pentatominae) por Rolston \& McDonald (1979). Posteriormente, Rolston (1992) forneceu chave e diagnoses para os gêneros de Ochlerini, incluindo novas sinonímias, descreveu nove novos gêneros com 11 novas espécies, passando a tribo a contar com 101 espécies descritas em 28 gêneros. Após os trabalhos de CAMPos \& Grazia (2000, 2001), CAMPos et al. (2004), ORTEGa-LeON \& THOMAs (2004) e CAMPos (2005), este número foi elevado para 30 gêneros e 107 espécies. As espécies de Ochlerini estão distribuídas do México à Argentina, sendo a maioria dos gêneros (17) representados exclusivamente na América do Sul.

Pelo menos dois gêneros de Ochlerini, Lincus Stål, 1867 e Macropygium Spinola, 1837, incluem espécies que agem como vetores de tripanossomatídeos flagelados (Phytomonas spp.) em palmeiras e coqueiros na América do Sul, sendo importantes do ponto de vista fitossanitário (Desmier-de-Chenon, 1984; Couturier \& Kahn, 1989; Llosa et al., 1990; Dollet et al., 1993).

Dos 30 gêneros de Ochlerini, 14 são monotípicos: Barola Rolston, 1992, Brachelytron Ruckes, 1958, Clypona Rolston, 1992, Eritrachys Ruckes, 1959, Forstona Rolston, 1992, Herrichella Distant, 1911, Macropygium, Ocellatocoris Campos \& Grazia, 2001, Orbatina Ruckes, 1961, Parochlerus Breddin, 1904, Pseudadoxoplatys Rolston, 1992, Similiforstona Campos \& Grazia, 2000, Tetrochlerus Breddin, 1904 e Uvaldus Rolston, 1992. Destes, são conhecidos macho e fêmea de Eritrachys, Macropygium, Orbatina, Parochlerus e Pseudadoxoplatys; os demais gêneros têm apenas as fêmeas descritas e, com a exceção de Clypona e Herrichella, sendo conhecidos tão somente os holótipos.

A proposição de Ochlerini (RoLSTON, 1981) foi feita 
empiricamente com base em um único estado de caráter, terceiro artículo metatarsal das fêmeas com uma depressão dorsal; sua inclusão em Discocephalinae está baseada, também empiricamente, em um caráter, posição de inserção do rostro relativa ao nível da margem anterior dos olhos, que apresenta variação entre os gêneros da tribo.

O monofiletismo de Ochlerini e sua inclusão em Discocephalinae nunca foram testados. Apesar de existir um estudo cladístico para as superfamílias de Pentatomomorpha (HENRY, 1997) e um estudo para famílias e subfamílias de Pentatomoidea estar em andamento, até o presente poucos trabalhos foram realizados utilizando a metodologia cladística para analisar as relações filogenéticas na família (AHMAD \& KHAN, 1983; SCHAEFER \& AhMAD, 1987; GAPUd, 1991; HasAn \& Kitching, 1993; GraZia, 1997; BARCELlos \& GraZia, 2003; ForTES \& GRAZIA, 2005). Da mesma forma são poucos os estudos biogeográficos em Pentatomidae (GRAZIA, 1997; THOMAS, 2000; BARCELlos \& GRAZIA, 2003).

No presente trabalho é apresentada uma análise cladística dos gêneros de Ochlerini, bem como considerações biogeográficas de grupos monofiléticos resultantes da análise.

\section{MATERIAL E MÉTODOS}

Para a análise cladística foram observados, para 40 táxons terminais, 63 caracteres (Tab. I), sendo os 34 primeiros referentes à morfologia geral e os 29 últimos à morfologia de genitália; estes caracteres foram organizados em uma matriz de dados (Tab. II) para posterior análise. As autapomorfias dos táxons terminais foram desconsideradas na análise dos caracteres e não estão apresentadas, pois 17 dos 34 táxons terminais de Ochlerini são monotípicos e tais apomorfias seriam, na verdade, das espécies. Para os demais 17 táxons terminais, consideramos suficiente para sua sustentação a heterobatmia gerada na análise cladística. Todos os caracteres foram tratados como não-ordenados e polarizados segundo o método de comparação com o grupo-externo (Watrous \& WheEler, 1981; MAdDison et al., 1984). Foram selecionados como grupo-externo Marghita Ruckes, 1964 e Stictochilus Bergroth, 1918 (Pentatomini). Janeirona Distant, 1911 (Pentatomini), Dalpada Amyot \& Serville, 1843, Brochymena Amyot \& Serville, 1843 (Halyini) e Discocephala Laporte, 1833 (Discocephalini) foram incluídos no grupo-interno com a finalidade de testar sua posição em relação a Ochlerini.

A avaliação dos caracteres para a análise cladística foi realizada observando-se o maior número possível de espécies de cada gênero. Ao menos a espécie-tipo de cada gênero foi observada e, quando não estavam disponíveis holótipos ou parátipos, foram examinados espécimes identificados presentes nas coleções. Foram examinadas 56 espécies disponíveis nas coleções acessadas, do total de 107 espécies descritas. Destas, foi possível a dissecção da genitália da fêmea de 43 espécies pertencentes a 21 gêneros e da genitália do macho de 36 espécies pertencentes a 20 gêneros (Tab. III). Dados de literatura complementaram a avaliação dos caracteres para as demais espécies. O único gênero do qual não foi possível examinar nenhum espécime foi o monotípico
Herrichella, assim os estados de caracteres foram avaliados apenas a partir da literatura.

Os táxons terminais foram representados por gêneros quando todas as espécies analisadas não apresentaram variação nos estados de caracteres. Devido à necessidade de acomodar variações intragenéricas observadas, os gêneros Ochlerus Spinola, 1837, Stalius Rolston, 1992 e Alitocoris Sailer, 1950 foram divididos nos grupos: Ochlerus handlirschi Breddin, 1910; Ochlerus "a", que inclui as espécies com fêmeas apresentando os gonocoxitos 8 íntegros, exceto $O$. handlirschi; Ochlerus "b", que inclui as espécies com fêmeas apresentando os gonocoxitos 8 fendidos; Stalius trisinuatus Rolston, 1992 e Stalius "a", o último incluindo a espécie-tipo $S$. tartareus (Stål, 1862) e S. castaneus (Distant, 1893); Alitocoris parvus (Distant, 1880) e Alitocoris "a", que inclui as demais espécies. A matriz de dados foi construída com o auxílio do programa NEXUS 0.5.0 (PAGE, 2001). A análise cladística foi efetuada com o programa PAUP*4.0b10 (Swofford, 2003). Os cladogramas foram calculados por meio de análises heurísticas, com seqüência de adições ao acaso, retenção de três árvores a cada etapa da adição e 100 replicações, com o uso posterior do método de aproximações por pesagens sucessivas (FARRIS, 1969; CARPENTER, 1988, 1994). Os cladogramas de consenso estrito resultantes das análises antes e após a pesagem sucessiva foram calculados e comparados posteriormente para a verificação de grupos monofiléticos coincidentes, utilizando o programa WinClada 1.00.08 (NIXON, 2002). Nos resultados e na discussão foram adotadas as notações "grupo"” e "grupo*" (АмORIM, 1982, 1994). A terminologia das peças genitais seguiu Dupuis $(1955,1970)$.

Para a análise biogeográfica foram construídos cladogramas de área para grupos monofiléticos obtidos nos cladogramas de consenso estrito, conforme método detalhado em Morrone \& CRISCI (1995), Humphries \& PAREnti (1999) e Morrone (2004).

\section{RESULTADOS E DISCUSSÃO}

Análise cladística. A análise inicial resultou em 45.965 cladogramas de 156 passos, com índice de retenção (IR) de 0,78 e índice de consistência (IC) de 0,42; o cladograma de consenso estrito, considerando apenas as transformações não-ambíguas dos caracteres, apresentou 215 passos, IR =0,64 e IC =0,31 (Fig. 1). Após a pesagem sucessiva dos caracteres, foram obtidos 156 cladogramas igualmente parcimoniosos ( $\mathrm{IR}=0,90, \mathrm{IC}$ $=0,65)$; o cladograma de consenso estrito (172 passos, $\mathrm{IR}=0,86, \mathrm{IC}=0,56)$, considerou apenas as transformações não-ambíguas dos caracteres (Figs. 2-4).

Os resultados das análises, independentemente do uso de pesagem sucessiva, sustentam a hipótese de que a tribo forma um grupo monofilético que é grupo-irmão de Discocephalini, ou seja, Discocephalinae sensu Rolston (1981) é um grupo monofilético (Figs. 1, 2). Além disso, Halyini+Ochlerini, Marghita+Janeirona+Halyini e Marghita+Janeirona+Ochlerini são grupos merofiléticos; esses resultados suportam todas as propostas feitas por RoLSTON \& MCDONALD (1979), que removeram Marghita, Janeirona e os gêneros de 
Tabela I. Caracteres e estados utilizados na análise cladística dos gêneros de Ochlerini (0, plesiomórfico; 1, 2, apomórficos).

Caracteres
1- Declividade da cabeça.
3- Ápices das jugas em relação ao clípeo (Fig. 11, cl, j).
4- Processos anteoculares (Fig. 12, pra).
5- Olhos.
6- Carena longitudinal posterior aos olhos.

7- Distância entre o limite interno do olho e o limite externo do ocelo (Fig. 12, doo).

8- Distância entre os ocelos e a margem posterior dos olhos (Fig. 12, dpo).

9- Largura do olho (Fig. 10, lo).

10- Relação entre a distância da margem posterior dos olhos à base da cabeça e o comprimento do olho (Fig. 10, dob, co). 11- Inclinação dos olhos em relação ao plano dorsal da cabeça (Figs. 15-17, io, pdc)

12- Origem aparente do primeiro artículo do rostro (Figs. 18-20, il).

13- Ápice do primeiro artículo do rostro.

14- Pseudartículo entre os artículos I e II do rostro.

15- Segundo artículo do rostro.

16- Ápice do rostro.

17- Número de artículos antenais.

18- Primeiro artículo antenal.

19- Textura dos artículos antenais II e III.

20- Pronoto anterior aos ângulos umerais.

21- Cicatrizes do pronoto.

22- Extensão da área evaporatória metapleural (Fig. 29, aet).

23- Extensão da área evaporatória mesopleural (Fig. 29, aes).

24- Contorno da margem lateral externa da área evaporatória metapleural.

25- Metasterno.

26- Metasterno.

27- Ápice do escutelo em relação ao cório (Figs. 31-34, ac, ae).

28- Fêmures anteriores.

29- Terceiro artículo tarsal das pernas posteriores do macho.

30- Terceiro artículo tarsal das pernas posteriores da fêmea.

31- Base do terceiro urosternito.

32- Terço mediano longitudinal ventral do abdome.

33- Distância dos tricobótrios abdominais dos segmentos IV e V em relação aos respectivos espiráculos (Figs. 42, 43, dte, es, tr).

\section{Estados}

[0] - acompanha a do pronoto (Fig. 7)

[1] - declivosa em relação ao pronoto (Fig. 8).

[0] - maior que 2/3 da distância interocular (Figs. 9-11).

[1] - aproximadamente igual à metade da distância interocular (Fig. 12).

[0] - separados pelo clípeo (Figs. 10, 11).

[1] - contíguos (Figs. 9, 12).

[0] - ausentes (Figs. 10, 11).

[1] - presentes, agudos (Figs. 9, 12).

[0] - não-pedunculados (Fig. 13).

[1] - pedunculados (Fig. 14, pe).

[0] - ausente (Fig. 13).

[1] - presente (Fig. 14, cr).

[0] - igual ou maior que a metade do diâmetro do ocelo (Figs. 9, 11, 12).

[1] - praticamente inexistente (Fig. 10).

[0] - igual ou maior que o diâmetro do ocelo (Figs. 9, 10, 12).

[1] - inexistente (Fig. 11).

[0] - igual ou menor que 1/3 da distância interocular (Figs. 9, 10, 12, dio).

[1] - igual ou maior que a metade da distância interocular (Fig. 11).

[0] - aproximadamente iguais (Figs. 9, 10, 12).

[1] - distância equivale no máximo à metade do comprimento (Fig. 11).

[0] - inexistente (Fig. 15).

[1] - inclinados para baixo (Fig. 16).

[2] - inclinados para cima (Fig. 17).

[0] - anterior à margem anterior dos olhos (Figs. 18, 19, 1to).

[1] - posterior à margem anterior dos olhos (Fig. 20).

[0] - não atinge o prosterno (Fig. 19).

[1] - atinge o prosterno (Figs. 18, 20).

[0] - ausente (Figs. 19, 20).

[1] - presente (Fig. 18, air).

[0] - atinge ou ultrapassa as mesocoxas (Fig. 8, II).

[1] - entre pró- e mesocoxas (Fig. 7).

[0] - ultrapassa a metade do urosternito IV (Fig. 8, IV).

[1] - atinge no máximo a margem posterior do urosternito III (Fig. 7).

[0] - cinco (Figs. 21, 23).

[1] - quatro (Fig. 24).

[0] - largura equivalente a $1 / 3$ do comprimento (Fig. 21).

[1] - largura equivalente a $1 / 5$ do comprimento (Figs. 23, 24).

[2] - largura equivalente a $1 / 2$ do comprimento (Fig. 22).

[0] - lisa (Figs. 22-24).

[1] - com tubérculos diminutos (Fig. 21).

[0] - declivoso (Fig. 25).

[1] - não-declivoso (Fig. 26).

[0] - aproximadamente planas (Fig. 27).

[1] - com um tubérculo interno (Fig. 28, tc).

[0] - 2/3 da largura da metapleura (Fig. 29, mt).

[1] - 1/2 da largura da metapleura (Fig. 30).

[0] - ocupa quase a metade posterior da mesopleura (Fig. 29, ms).

[1] - ocupa no máximo uma estreita faixa junto ao limite entre meso- e metapleura (Fig. 30).

[0] - sinuosa (Fig. 29, mle).

[1] - sub-retilínea (Fig. 30).

[0] - elevado em relação às pleuras.

[1] - não-elevado em relação às pleuras.

[0] - não-carenado.

[1] - carenado.

[0] - não alcança o ápice do cório (Fig. 31).

[1] - alcança o ápice do cório (Fig. 32).

[2] - ultrapassa o ápice do cório (Fig. 33).

[0] - inermes (Fig. 35).

[1] - armados com espinhos (Fig. 36, ef).

[0] - dorsalmente convexo.

[1] - dorsalmente sulcado.

[0] - dorsalmente convexo (Fig. 37).

[1] - dorsalmente aplainado (Fig. 38).

[2] - dorsalmente sulcado (Fig. 39, s).

[0] - com tubérculo (Fig. 40, ta).

[1] - sem tubérculo (Fig. 41).

[0] - convexo.

[1] - sulcado.

[0] - equivale ao diâmetro do espiráculo (Fig. 42, de).

[1] - equivale a duas vezes ou mais o diâmetro do espiráculo (Fig. 43). 
Continuação Tabela I.

34- Glândulas de cera abdominais.

35- Expansões lobulares dos ângulos póstero-laterais do pigóforo.

36- Projeção mediana cônica do bordo ventral do pigóforo.

37- Superfície dorsal do segmento $\mathrm{X}$ dos machos.

38- Projeção pós-anal ventral no segmento $\mathrm{X}$ dos machos.

39- Área enrijecida no segmento X dos machos ventralmente ao ânus.

40- Membrana articular junto à margem posterior do sétimo urotergito dos machos (Figs. 52, 53, m).

41- Margem posterior dos gonocoxitos 8 .

42- Sutura longitudinal mediana do gonocoxito 9

43- Gonocoxito 9.

44- Laterotergitos 9 ao longo das margens medianas.

45- Laterotergitos 9.

46- Segmento X da fêmea.

47-Gonapófise 9 no terço lateral.

48- Chitinellipsen.

49- Calibre do ductus receptaculi anterior à área vesicular.

50- Parede intermediária da área vesicular.

51- Parede interna da área vesicular.

52- Comprimento somado da pars intermedialis e capsula seminalis (Fig. 61, cs, pi).

53- Forma da pars intermedialis.

54- Comprimento da pars intermedialis.

55- Forma da capsula seminalis.

56- Inserção dos dentes na capsula seminalis (Figs. 62-65, dc).

57- Espessura dos dentes da capsula seminalis (Figs. 62-65, dc).

58- Comprimento da vésica em relação à phallotheca (Figs. 66-69, ph, v).

59- Base da vésica junto à phallotheca.

60- Vésica.

61- Eixo longitudinal da vésica.

62- Vésica nos 2/3 apicais.

63- Abertura apical da vésica.
[0] - ausentes.

[1] - presentes.

[0] - ausentes (Fig. 44).

[1] - presentes (Fig. 45, el).

[0] - ausente (Fig. 46).

[1] - presente (Fig. 47, cbv).

[0] - de textura uniforme, rígida (Fig. 44, X).

[1] - membranosa pelo menos no terço basal (Fig. 45, X).

[0] - ausente (Fig. 48).

[1] - presente (Fig. 49).

[0] - ausente (Fig. 50).

[1] - presente (Fig. 51, esa).

[0] - em forma de crescente (Fig. 52).

[1] - expandida posteriormente na região mediana (Fig. 53).

[0] - não-projetada, no máximo ligeiramente túmida (Fig. 55).

[1] - projetada, formando um assoalho (Fig. 54, gc8).

[0] - ausente (Fig. 56).

[1] - presente (Fig. 57, sgc9).

[0] - exposto (Figs. 56, 57, gc9).

[1] - oculto pelos gonocoxitos 8 (Fig. 58)

[0] - afastados (Fig. 56, la9).

[1] - tocando-se (Figs. 57, 58).

[0] - livres (Fig. 59, la9).

[1] - fusionados ao gonocoxito 9 (Fig. 60).

[0] - exposto (Figs. 56, 57, X).

[1] - oculto pelos laterotergitos 9 (Fig. 58).

[0] - lisa (Figs. 59, 60, g9).

[1] - com dobras recobertas de microestruturas cônicas (Fig. 61, vg9)

[0] - presentes (Figs. 59-61, ch).

[1] - ausentes.

[0] - pelo menos três vezes menor que o calibre da parede externa da área vesicular (Figs. 59, 60, dr, av).

[1] - de mesmo calibre que a parede externa da área vesicular (Fig. 61, te).

[0] - de calibre uniforme (Fig. 60).

[1] - alargada na base (Figs. 59, 61, tm).

[0] - de calibre uniforme (Figs. 59, 60).

[1] - estreitando-se em direção à pars intermedialis (Fig. 61, ti).

[0] - menor do que $1 / 3$ do comprimento da área vesicular (Fig. 60).

[1] - aproximadamente a metade do comprimento da área vesicular

(Figs. 59, 61)

[0] - cilíndrica (Figs. 59, 61, pi).

[1] - alargada na região mediana (Figs. 60, 62, 63, 65, pi).

[0] - no máximo o dobro da capsula seminalis (Figs. 62, 63).

[1] - pelo menos quatro vezes mais longa que a capsula seminalis

(Fig. 64, cs).

[0] - sem constrição mediana (Figs. 62, 65).

[1] - com constrição mediana (Fig. 64, cs).

[0] - próximo à base da capsula seminalis (Figs. 62, 64).

[1] - no ápice da capsula seminalis (Fig. 65).

[0] - esguios, no máximo 1/3 da largura da capsula seminalis (Figs. 62, $64,65)$.

[1] - robustos, quase tão largos quanto a capsula seminalis (Fig. 63).

[0] - no máximo de mesmo comprimento que a phallotheca (Fig. 66).

[1] - pelo menos 1,5 vez mais longa que a phallotheca (Figs. 67, 69).

[0] - sem projeções dorso-laterais (Figs. 66, 67, 69).

[1] - com projeções alares dorso-laterais (Fig. 68, pav).

[0] - não-articulada (Figs. 66, 68, 69).

[1] - articulada (Fig. 67, av).

[0] - posicionado ao longo do plano longitudinal (Figs. 66, 68, 69).

[1] - retorcido em hélice, projetando-se lateralmente ao plano longitudinal (Fig. 67).

[0] - livre (Figs. 66-68).

[1] - circundada por membrana da conjuntiva (Fig. 69).

[0] - circular (Fig. 66).

[1] - chanfrada (Fig. 67, aav).
Ochlerini da tribo Halyini. Entretanto, o merofiletismo de Pentatominae é sugerido, já que Janeirona+Halyini não constitui um grupo monofilético. Apenas através de estudos cladísticos abrangentes de Pentatomidae será possível aproximar-se melhor das corretas relações filogenéticas entre as subfamílias e tribos, bem como sua composição.
Nos cladogramas de consenso (Figs. 1, 2), Discocephala+Ochlerini apresenta como sinapomorfias o segmento basal do rostro alongado $\left(13_{1}\right)$ e o segmento $\mathrm{X}$ dos machos dorsalmente membranoso no terço basal $\left(37_{1}\right)$. O primeiro estado de caráter foi citado por RoLSTON (1981) como diagnóstico para Ochlerini e não é conhecido 
para outros pentatomídeos neotropicais, exceto por alguns Edessinae que apresentam um encurtamento do rostro e declividade da cabeça, e parte de Asopinae, onde o rostro é extremamente robusto, alargado, adaptação ao hábito predador. $\mathrm{O}$ segundo estado de caráter não é conhecido para outros pentatomídeos neotropicais. A presença de metasterno carenado $\left(26_{1}\right)$ é uma terceira sinapomorfia para as tribos de Discocephalinae, com reversões em Stalius trisinuatus, Stalius "a" e Lincus. Muitos gêneros de Pentatomidae apresentam este estado de caráter e seu papel na filogenia do grupo ainda é desconhecida. O clado de Ochlerini, reunindo Adoxoplatys + Neoadoxoplatys e Pseudadoxoplatys ${ }^{+}$, é suportado por uma sinapomorfia, o terceiro artículo metatarsal das fêmeas dorsalmente aplainado $\left(30_{1}\right)$.

Na primeira dicotomia observada em Ochlerini, é estabelecido o parentesco dos grupos Adoxoplatys + Neoadoxoplatys e Pseudadoxoplatys ${ }^{+}$. O primeiro grupo compartilha a presença de olhos inclinados para baixo $\left(11_{1}\right)$ e de um falso artículo rostral entre os dois primeiros artículos $\left(14_{1}\right)$, além de três homoplasias: pronoto plano $\left(20_{1}\right)$, homoplástico com Alathetus Dallas, 1851, fêmures anteriores com espinhos ventrais $\left(28_{1}\right)$, homoplástico com Paralincus, e vésica mais longa que a phallotheca $\left(58_{1}\right)$, homoplástico com Eritrachys, Catulona Rolston, 1992, Alitocoris e o grupo Miopygium ${ }^{+}$. A respeito do último caráter, GAPUD (1991) comparou o comprimento da vésica ao comprimento da conjuntiva, estrutura ausente em muitos Discocephalinae. Hasan \& Kitching (1993) consideraram que vésica curta seria uma plesiomorfia e longa seria uma apomorfia. O segundo grupo, Pseudadoxoplatys ${ }^{+}$, é suportado pela presença de expansões nos ângulos póstero-laterais do pigóforo $\left(35_{1}\right)$, presença de uma área subanal espessada e rígida (39), fêmeas apresentando gonocoxito 9 com sutura longitudinal, com múltiplas reversões $\left(42_{1}\right)$ e laterotergitos 9 tocando-se medianamente $\left(44_{1}\right)$, com reversão em Coranda Rolston, 1992, e machos com vésica apresentando abertura em chanfro $\left(63_{1}\right)$.

As relações internas do grupo Ocellatocoris* são pouco resolvidas (Fig. 1). Este grupo, entretanto, é suportado pela presença de área evaporatória metapleural reduzida $\left(22_{1}\right)$, estado observado por HASAN \& KITCHING (1993) nas tribos Strachini, Asopini e Podopini; área evaporatória mesopleural reduzida $\left(23_{1}\right)$; presença de sulco dorsal no terceiro artículo metatarsal das fêmeas $\left(30_{2}\right)$, estado observado por STÅL (1867) e citado por Rolston $(1981,1992)$ como a principal característica diagnóstica de Ochlerini; segmento $\mathrm{X}$ das fêmeas oculto pelos laterotergitos 9 (46. $)$, com reversões em Coranda, Schraderiellus Rider, 1998, S. trisinuatus, Paralincus, Uvaldus e Clypona+Macropygium.

Após a pesagem sucessiva dos caracteres (Figs. 2-4), Ocellatocoris* ficou composto por dois grandes grupos monofiléticos: Ocellatocoris ${ }^{+}$(Fig. 3), que inclui Moncus+O. handlirschi como grupo apical, e o clado contendo os grupos-irmãos Eritrachys + Phereclus e Herrichella* (Fig. 4). O grupo Ocellatocoris ${ }^{+}$compartilha $^{*}$ a presença de escutelo longo, alcançando o ápice do cório $\left(27_{1}\right)$ com uma reversão em Coranda, sendo que, em outras subfamílias e tribos de Pentatomidae, alguns gêneros apresentam o escutelo muito desenvolvido ( $\mathrm{p}$.

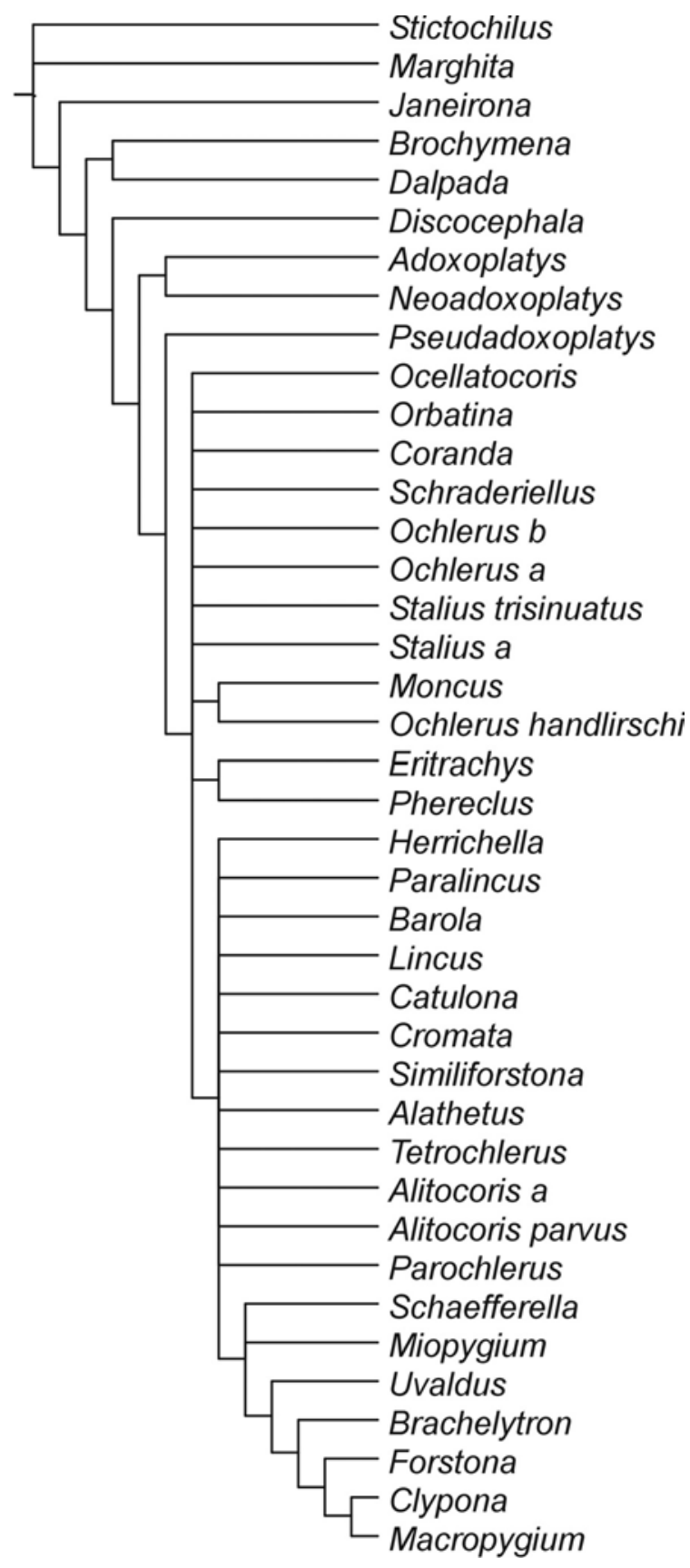

Fig. 1. Cladograma de consenso estrito para os gêneros de Ochlerini, anterior à pesagem sucessiva (215 passos, $\mathrm{IR}=0,64, \mathrm{IC}=0,31)$.

ex., Graphosoma Laporte, 1832, Podopinae; Discocera Laporte, 1833, Asopinae e Antiteuchus Dallas, 1851, Discocephalini). Ocellatocoris ${ }^{+}$inclui os seguintes grupos monofiléticos: Orbatina ${ }^{+}$, suportado pela pequena distância entre a margem posterior dos olhos e a base da cabeça $\left(10_{1}\right)$, estado homoplástico com Discocephala e comparável ao que Rolston (1992) descreveu como afastamento dos olhos em relação ao pronoto, tendo considerado o maior afastamento como um estado derivado que reuniria os gêneros Barola, Eritrachys, Herrichella, Paralincus e Phereclus Stål, 1862; Corand $a^{+}$, suportado pela grande largura dos olhos $\left(9_{1}\right)$; Schraderiellus ${ }^{+}$, suportado por ocelos posicionados 
Tabela II. Matriz polarizada de dados para a análise cladística dos 30 gêneros de Ochlerini (0, plesiomorfia; 1, 2, apomorfia; ?, caráter não observado; -, caráter não comparável).

\begin{tabular}{|c|c|c|c|c|c|c|c|}
\hline \multirow[b]{3}{*}{ Táxon } & \multicolumn{6}{|c|}{ Caráter/Estado } & \multirow[b]{2}{*}{666} \\
\hline & 0000000001 & 1111111112 & 2222222223 & 3333333334 & 4444444445 & 5555555556 & \\
\hline & 1234567890 & 1234567890 & 1234567890 & 1234567890 & 1234567890 & 1234567890 & 123 \\
\hline Stictochilus & 0010000000 & 0000000000 & 0000000000 & 0000000000 & 0000000000 & 0000000000 & 000 \\
\hline Marghita & 0000000000 & 0000000000 & 0000000000 & 0000000000 & 0000000000 & 0010000000 & 000 \\
\hline Janeirona & 0000000000 & 0000000000 & 0000000000 & 0100000000 & 0000000000 & 0000000000 & 000 \\
\hline Brochymena & 0000000000 & 0000000000 & 0000100000 & 1101000000 & 0000000000 & 0000000000 & 000 \\
\hline Dalpada & 0000000000 & 0000110000 & 0000100000 & 1101000000 & 0000000000 & 0000000000 & 000 \\
\hline Discocephala & 0110000101 & 0110000000 & 0000110000 & 1100001000 & 0000000000 & 0000000000 & 000 \\
\hline Adoxoplatys & 0000000000 & 1011000011 & 0000010101 & 0110001000 & 0000000001 & 0100000100 & 000 \\
\hline Neoadoxoplatys & 0000000000 & 1011000011 & 0000110101 & 1100001000 & 0000000000 & 0000000100 & 000 \\
\hline Pseudadoxoplatys & 0000000000 & 0010000210 & 0000110011 & 1010101010 & 0101000000 & 0000001000 & 001 \\
\hline Eritrachys & 1000100000 & 2110000210 & 1110110012 & 1110001010 & 0101010100 & 0010000100 & 001 \\
\hline Phereclus & 1000100000 & 2010000210 & $11101100 ? 2$ & $1110 ? ? ? ? ? ?$ & 0101010101 & $0000001 ? ? ?$ & $? ? ?$ \\
\hline Ocellatocoris & 0000000000 & 0010000000 & $01101110 ? 2$ & $1110 ? ? ? ? ? ?$ & $010101 ? ? ? ?$ & $? ? ? ? ? ? ? ? ? ?$ & $? ? ?$ \\
\hline Orbatina & 0000000001 & 0010000200 & 0110111002 & 1110101010 & $0-0101 ? ? ? ?$ & ???????000 & 001 \\
\hline Coranda & 0000000011 & 0010000200 & $01101100 ? 2$ & 1110101010 & $000000 ? ? ? ?$ & ?????????? & $? ? ?$ \\
\hline Schraderiellus & 0000000111 & 0010000200 & 0110111002 & 1110101010 & 0001100000 & 0001000000 & 001 \\
\hline Ochlerus b & 0000000111 & 0010000200 & 0110111002 & 1110101010 & 0101110000 & 0010000000 & 001 \\
\hline Ochlerus a & 0000000111 & 0010000200 & 0110111002 & 1110101010 & 0101110000 & 0010000010 & 001 \\
\hline Stalius trisinuatus & 0000000111 & 0010000200 & 0110101002 & 1010101010 & 0001100000 & 0010010010 & 001 \\
\hline Stalius a & 0000000111 & 0010000200 & 0110102002 & 1110101010 & 0011110000 & 0010010010 & 001 \\
\hline Moncus & 0000000111 & 0010000200 & 0110112012 & 1110101010 & 0001110001 & 0010010010 & 001 \\
\hline Ochlerus handlirschi & 0000000111 & 0010000200 & 0110112012 & 1110101010 & 0001110000 & 0010010010 & 001 \\
\hline Brachelytron & 0011000000 & 0110010100 & 011111-0?2 & $1110 ? ? ? ? ? ?$ & $010101 ? ? ? ?$ & ?????????? & $? ? ?$ \\
\hline Herrichella & 0000110000 & $? 110010200$ & $011 ? 1100 ? 2$ & $1110 ? ? ? ? ? ?$ & ?????????? & ?????????? & $? ? ?$ \\
\hline Paralincus & 0000110000 & 0110000010 & 1111110112 & 1110101011 & 0111000000 & 0001000000 & 001 \\
\hline Barola & 0000111000 & 0110000000 & $01111100 ? 2$ & $1110 ? ? ? ? ? ?$ & $010101 ? ? ? ?$ & ?????????? & $? ? ?$ \\
\hline Lincus & 0000111000 & 0110000000 & 0110100012 & 1110001010 & 0001010000 & 0001000000 & 001 \\
\hline Catulona & 0000111000 & 0110000000 & 0111110012 & 1110001011 & 0111010000 & 0001100100 & 001 \\
\hline Cromata & 0000111000 & 0110000000 & 0111110012 & 1110001011 & 0101010000 & $0000001 ? ? ?$ & $? ? ?$ \\
\hline Similiforstona & 0000110000 & 0010011000 & $01111100 ? 2$ & $1110 ? ? ? ? ? ?$ & $110101 ? ? ? ?$ & $? ? ? ? ? ? ? ? ? ?$ & $? ? ?$ \\
\hline Alathetus & 0001110000 & 0110001201 & 0111110012 & 1110011010 & 0101011001 & 0001100101 & 101 \\
\hline Tetrochlerus & 0000110000 & 0110001000 & $01111100 ? 2$ & $1110 ? ? ? ? ? ?$ & $0101 ? 1 ? ? ? ?$ & ?????????? & $? ? ?$ \\
\hline Alitocoris a & 0000110000 & 0110000000 & 0111110012 & 1110101010 & 0101011011 & 0101000101 & 001 \\
\hline Alitocoris parvus & 0111110000 & 0110000200 & 0111110012 & 1110011010 & 0001011001 & 1001010100 & 101 \\
\hline Parochlerus & 0000110000 & 0110000000 & $01111100 ? 2$ & 1110101110 & 0101011001 & $010100000 ?$ & 001 \\
\hline Schaefferella & 0011110000 & 0110000000 & 0111110012 & 1010101110 & 0101011011 & 0101000001 & 101 \\
\hline Miopygium & 0111110000 & 0110111000 & 0111110012 & 1010101111 & 0101011011 & 0101100101 & 101 \\
\hline Uvaldus & 0011110000 & 0110010000 & 0111110012 & 1010111110 & 0101001111 & 1101000100 & 011 \\
\hline Forstona & 0011110000 & 0110110100 & $01111100 ? 2$ & $1010 ? ? ? ? ? ?$ & $110101 ? ? ? ?$ & ?????????? & $? ? ?$ \\
\hline Clypona & 0111110000 & 0110110100 & $01111100 ? 2$ & $1010 ? ? ? ? ? ?$ & 0101001011 & $1101000 ? ? ?$ & $? ? ?$ \\
\hline Macropygium & 0111110000 & 0110110100 & 0111110012 & 1010111110 & $010100101 ?$ & 1101000100 & 111 \\
\hline
\end{tabular}

próximos ao nível posterior dos olhos $\left(8_{1}\right)$, homoplástico com Discocephala, e pela fusão parcial entre os laterotergitos 9 e gonocoxito $9\left(45_{1}\right)$; Ochlerus $\mathrm{b}^{+}$, suportado pela pars intermedialis alargada na região mediana (53.), homoplástico com Eritrachys e Marghita; Ochlerus $\mathrm{a}^{+}$, suportado pelas projeções alares dorsolaterais na vésica $\left(59_{1}\right)$; Stalius trisinuatus ${ }^{+}$, suportado pelos dentes inseridos no ápice da capsula seminalis (56), homoplástico com A. parvus e encontrado em parte de Discocephalini e em outros gêneros de Pentatomidae (p. ex., Caura Stål, 1865); Stalius $\mathrm{a}^{+}$, suportado pelo escutelo muito longo, ultrapassando o nível do ápice do cório $\left(27_{2}\right)$; Moncus $+O$. handlirschi, o grupo apical, é suportado por uma homoplasia, terceiro artículo metatarsal dos machos sulcado (291), homoplástico com Pseudadoxoplatys $^{+}$. Rolston (1992) considerou que os machos de Adoxoplatys Breddin, 1903 e Neoadoxoplatys apresentam um aplainamento do terceiro artículo dos metatarsos, entretanto nossas observações não suportam tal afirmação.

O clado reunindo Eritrachys + Phereclus e

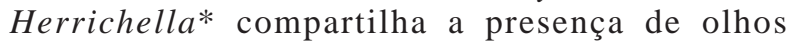
pedunculados $\left(5_{1}\right)$, com reversão em Brachelytron; e uma homoplasia, vésica longa $\left(58_{1}\right)$. Eritrachys + Phereclus é suportado pela deflexão da cabeça em relação ao pronoto $\left(1_{1}\right)$ e pela inclinação dos olhos para cima $\left(11_{2}\right)$, além de dois estados homoplásticos, cicatrizes do pronoto com um tubérculo interno $\left(21_{1}\right)$ e fêmeas desprovidas de chitinellipsen $\left(48_{1}\right)$; Herrichella* compartilha a presença de carena longitudinal látero-ventral posterior aos olhos $\left(6_{1}\right)$, estado não registrado para outros pentatomídeos neotropicais; margem lateral da área evaporatória metapleural sub-retilínea $\left(24_{1}\right)$ e fêmeas apresentando pars intermedialis pelo menos quatro vezes mais longa que a capsula seminalis $\left(54_{1}\right)$, homoplástico com Schraderiellus. 
Tabela III. Lista das espécies de Ochlerini. As espécies examinadas são aquelas com a indicação, entre parênteses, das instituições de procedência do material (conforme ARnETT et al., 1993). As espécies-tipo e as espécies dissecadas estão indicadas.

\begin{tabular}{|c|c|c|c|}
\hline \multirow[t]{2}{*}{ Gênero } & \multirow[t]{2}{*}{ Espécie } & \multicolumn{2}{|c|}{ Dissecção da genitália } \\
\hline & & Fêmea & Macho \\
\hline Adoxoplatys Breddin, 1903 & $\begin{array}{l}\text { A. brasiliensis Kormilev, } 1951 \\
\text { A. bridarollii Kormilev, } 1949 \text { (MACN) } \\
\text { A. comis Breddin, } 1903 \text { (AMNH, CASC, MACN) } \\
\text { A. gallardoi Kormilev, } 1951 \text { (MACN, UFRG) } \\
\text { A. giaii Kormilev, } 1950 \text { (AMNH, MACN, UFRG) } \\
\text { A. minax Breddin, } 1903 \text { (tipo) (UFRG) } \\
\text { A. panamensis Kormilev, 1951 } \\
\text { A. willineri Kormilev, } 1949 \text { (AMNH, MACN, MPEG) }\end{array}$ & $\begin{array}{l}X \\
X \\
X \\
X \\
X\end{array}$ & $\begin{array}{l}X \\
X \\
X \\
X\end{array}$ \\
\hline Alathetus Dallas, 1851 & $\begin{array}{l}\text { A. haitiensis Rolston, } 1982 \text { (AMNH) } \\
\text { A. rufitarsis Dallas, } 1851 \text { (tipo) (AMNH, BPBM) }\end{array}$ & $\mathrm{X}$ & $\mathrm{X}$ \\
\hline Alitocoris Sailer, 1950 & $\begin{array}{l}\text { A. brunneus Sailer, } 1950 \text { (AMNH, DBTC) } \\
\text { A. maculosus Sailer, } 1950 \\
\text { A. manni Sailer, } 1950 \text { (AMNH, DBTC, IZAV) } \\
\text { A. parvus Sailer, } 1950 \text { (AMNH, DBTC, INBio, IZAV) } \\
\text { A. schraderi Sailer, } 1950 \text { (tipo) (AMNH, DBTC, INBio) }\end{array}$ & $\begin{array}{l}X \\
X \\
X \\
X\end{array}$ & $\begin{array}{l}X \\
X\end{array}$ \\
\hline Barola Rolston, 1992 & B. farfala Rolston, 1992 (tipo) (FMNH) & & \\
\hline Brachelytron Ruckes, 1958 & B. angelicus Ruckes, 1958 (tipo) (AMNH) & & \\
\hline Catulona Rolston, 1992 & $\begin{array}{l}\text { C. apaga Rolston, } 1992 \text { (DZUP, UFRG, USNM) } \\
\text { C. lucida Campos \& Grazia, } 2004 \text { (MZSP, UFRG) } \\
\text { C. pensa Rolston, } 1992 \text { (tipo) (DZUP, MZSP, UFRG) }\end{array}$ & $\begin{array}{l}X \\
X\end{array}$ & $\begin{array}{l}X \\
X\end{array}$ \\
\hline Clypona Rolton, 1992 & C. aerata Rolston, 1992 (tipo) (AMNH, USNM) & $\mathrm{X}$ & \\
\hline Coranda Rolston, 1992 & $\begin{array}{l}\text { C. castana Rolston, } 1992 \\
\text { C. picipes (Stål, 1872) (tipo) (MUJ) }\end{array}$ & & \\
\hline Cromata Rolston, 1992 & $\begin{array}{l}\text { C. ornata Rolston, } 1992 \text { (tipo) (AMNH, CPJB, MNRJ, MZSP, UFRG) } \\
\text { C. graziae Campos, } 2005 \text { (INPA, IZAV) }\end{array}$ & $\mathrm{X}$ & \\
\hline Eritrachys Ruckes, 1959 & E. bituberculata Ruckes, 1959 (tipo) (DBTC, INBio, MICR, MZUF) & $\mathrm{X}$ & $\mathrm{X}$ \\
\hline Forstona Rolston, 1992 & F. speciosa Rolston, 1992 (tipo) (AMNH) & & \\
\hline Herrichella Distant, 1911 & H. thoracica Distant, 1911 (tipo) & & \\
\hline Lincus Stål, 1867 & $\begin{array}{l}\text { L. anulatus Rolston, } 1983 \text { (MZSP) } \\
\text { L. apollo Dolling, } 1984 \\
\text { L. armiger Breddin, } 1908 \\
\text { L. breddini Rolston, } 1983 \\
\text { L. convexus Rolston, } 1983 \\
\text { L. croupius Rolston, } 1983 \\
\text { L. dentiger Breddin, } 1904 \\
\text { L. discessus (Distant, } 1900 \text { ) } \\
\text { L. fatigus Rolston, } 1983 \\
\text { L. hebes Rolston, } 1989 \text { (UFRG) } \\
\text { L. incisus Rolston, } 1983 \text { (UFRG) } \\
\text { L. lamelliger Breddin, } 1908 \text { (AMNH) } \\
\text { L. laminatus Rolston, } 1983 \\
\text { L. lethifer Dolling, } 1984 \\
\text { L. leviventris Rolston, } 1983 \text { (MNRJ) } \\
\text { L. lobuliger Breddin, } 1908 \text { (CNPS, MCNZ, UFRG) } \\
\text { L. manchus Rolston, } 1983 \\
\text { L. malevolus Rolston, } 1989 \text { (AMNH) } \\
\text { L. modicus Rolston, } 1983 \\
\text { L. operosus Rolston, } 1983 \\
\text { L. parvulus (Ruckes, } 1958 \text { ) (AMNH) } \\
\text { L. repizcus Rolston, } 1983 \\
\text { L. rufospilotus (Westwood, 1837) (tipo) } \\
\text { L. securiger Breddin, } 1904 \text { (UFRG) } \\
\text { L. singularis Rolston, } 1983 \\
\text { L. sinuosus Rolston, } 1983 \\
\text { L. spathuliger Breddin, } 1908 \\
\text { L. spurcus Rolston, } 1989 \text { (UFRG) } \\
\text { L. styliger Breddin, } 1908\end{array}$ & $\begin{array}{l}X \\
X \\
X\end{array}$ & $\begin{array}{l}X \\
X\end{array}$ \\
\hline
\end{tabular}


Continuação Tabela III.

Macropygium Spinola, 1837

Miopygium Breddin, 1904

Moncus Stål, 1867

Neoadoxoplatys Kormilev, 1956

Ocellatocoris Campos \& Grazia, 2001

Ochlerus Spinola, 1837

Orbatina Ruckes, 1961

Paralincus Rolston, 1983

Parochlerus Breddin, 1904

Phereclus Stål, 1862

Pseudadoxoplatys Rolston, 1992

Schaefferella Spinola, 1850

Schraderiellus Rider, 1998

Similiforstona Campos \& Grazia, 2000

Stalius Rolston, 1992

Tetrochlerus Breddin, 1904

Uvaldus Rolston, 1992

Incertae sedis
L. substyliger Rolston, 1983

L. subuliger Breddin, 1908

L. tumidifrons Rolston, 1983

L. vallis Rolston, 1983 (IZAV, UFRG)

L. vandoesburgi Rolston, 1983

L. varius Rolston, 1983

M. reticulare (Fabricius, 1803) (tipo) (AMNH, BPBM, DBTC DZUP, MACN, MCNZ, MRSN)

M. cyclopeltoides Breddin, 1904 (tipo) (BPBM, DZUP, INPA, $\quad$ X $\quad$ X MZSP, UFRG)

M. grossa Ruckes, 1958 (DZPR, UFRG) $\quad$ X $\quad$ X

M. obscurus (Dallas, 1851) (tipo) (AMNH, DCMB, INBio, UFRG) X X $\quad$ X

M. monachus Bergroth, 1914

N. haywardi Kormilev, 1956

N. longirostra Ruckes, 1959

N. saileri Kormilev, 1956 (tipo) (AMNH, MACN)

O. dasys Campos \& Grazia, 2001 (tipo) (MNRJ)

O. bergrothi Breddin, 1910

O. bistillatus Breddin, 1910

O. cinctus Spinola, 1837 (tipo) (AMNH, MRSN, UFRG)

X

$\mathrm{X}$

O. communis Breddin, 1910

O. cotylophorus Breddin, 1910

O. dentijugis Breddin, 1910

O. handlirschi Breddin, 1910 (AMNH)

O. incisulus Breddin, 1910

O. notatulus Breddin, 1910

O. profanus Breddin, 1910

O. rusticus Breddin, 1910

O. signoreti Breddin, 1910

O. sordidus Herrich-Schäffer, 1844

O. stylulatus Breddin, 1910

O. tenuicornis Breddin, 1910 (UFRG)

$\mathrm{X}$

O. fuliginia Ruckes, 1961 (tipo) (AMNH)

$\mathrm{X}$

P. bimaculatus (Ruckes, 1958) (DBTC, MRSN, UFRG) X X X

$P$. silvae Rolston, 1983

P. terminalis (Walker, 1867) (tipo)

P. latus Breddin, 1904 (tipo) (AMNH)

P. pluto Stål, 1862 (tipo) (AMNH)

$P$. punctatus (Dallas, 1851)

P. antennatus Distant, 1911

P. mendacis Rolston, 1992 (tipo) (AMNH, CASC, MNRJ, UFRG) X

$\mathrm{X}$

S. fusca Rolston, 1992

S. incisa (Herrich-Schäffer, 1839) (tipo) (AMNH, DZUP, UFRG, MZSP) $\quad X \quad X$

cinctus (Ruckes, 1959) (AMNH, BPBM, DBTC, INBio)

hughesae (Ruckes, 1959) (tipo) (DBTC)

$\mathrm{X} \quad \mathrm{X}$

$\mathrm{X} \quad \mathrm{X}$

S. castaneus (Distant, 1893)

S. leonae Ortega, 2004

S. scutellatus Thomas, 2004

S. tartareus (Stål, 1862) (tipo) (DBTC, INBio, MICR)

S. trisinuatus Rolston, 1992 (DBTC, INBio)

$\begin{array}{ll}X & X \\ X & X\end{array}$

T. fissiceps Breddin, 1904 (tipo) (USNM)

U. concolor Rolston, 1992 (tipo) (CMNH, DZUP, UFRG) $\quad$ X $\quad$ X

Melanodermus dilutipes Breddin, 1904 


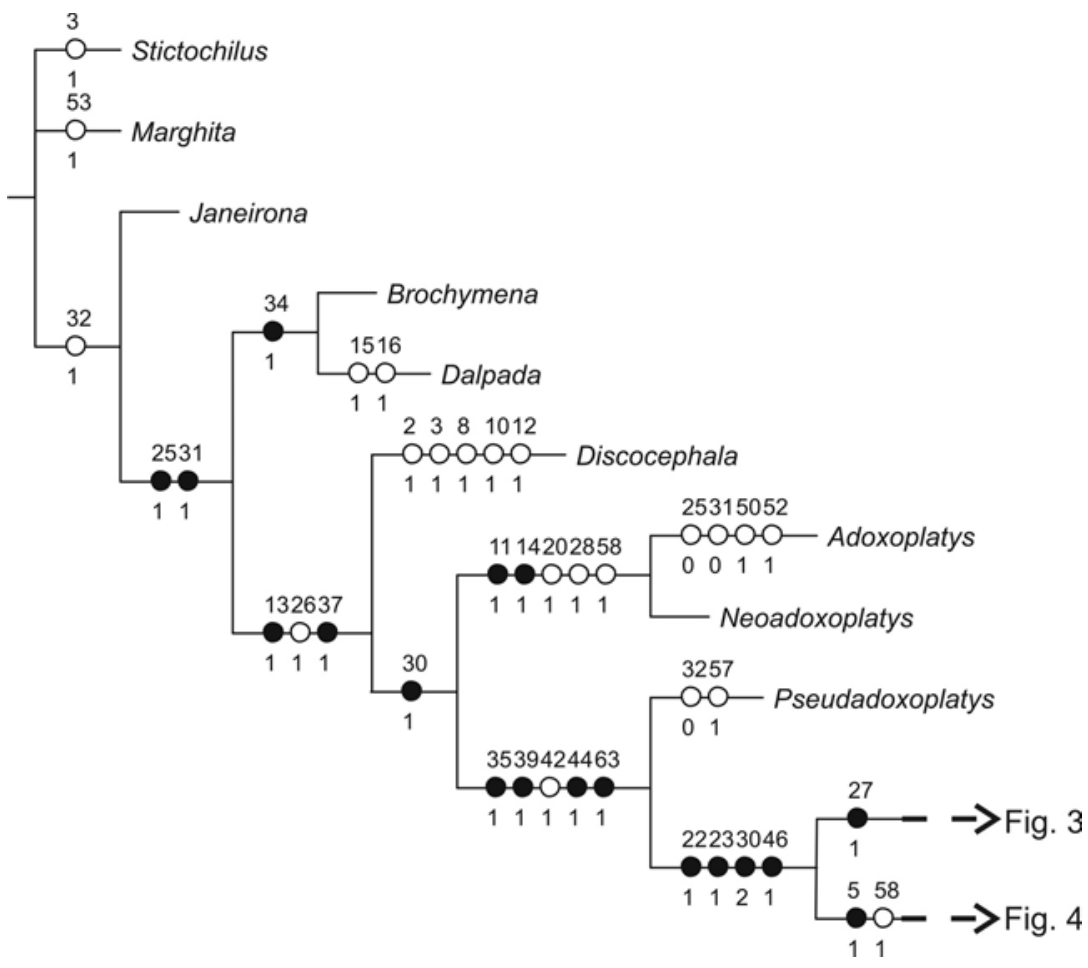

Fig. 2. Cladograma de consenso estrito para os gêneros de Ochlerini após a pesagem sucessiva $(172$ passos, IR $=0,86$, IC $=0,56)$. O clado do grupo Ocellatocoris ${ }^{+}$e o clado reunindo Eritrachys + Phereclus e Herrichella* não estão apresentados $(\mathbf{O}=$ sinapomorfia; $\mathbf{O}=$ homoplasia).

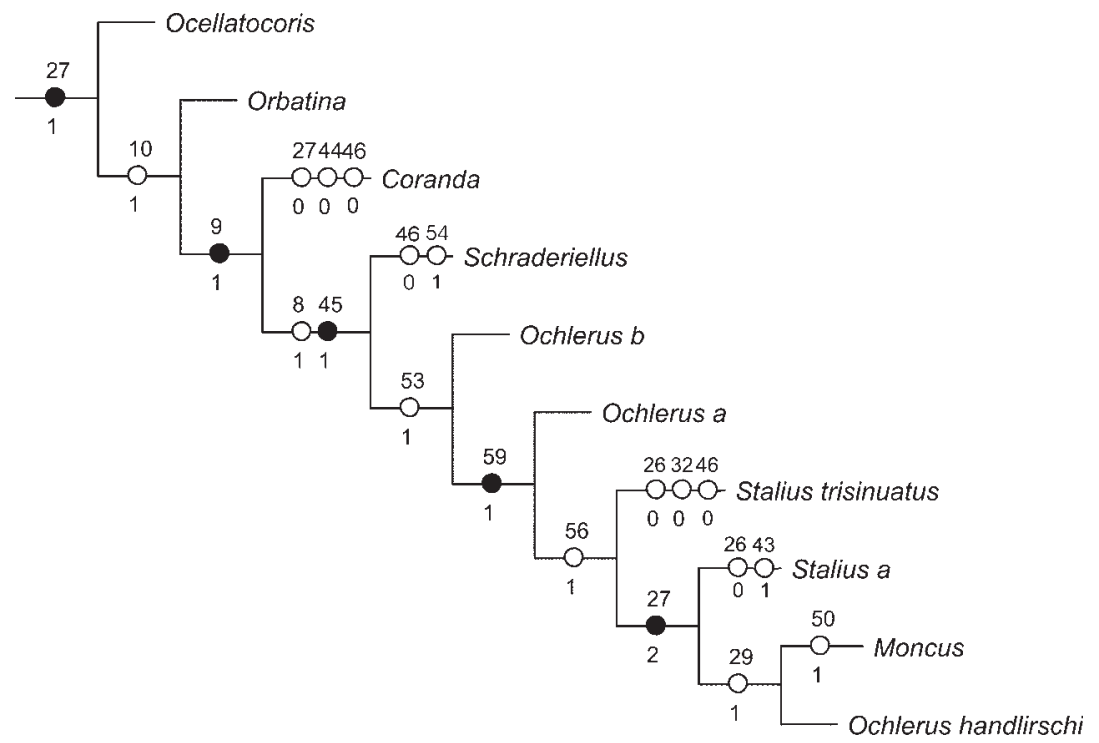

Fig. 3. Relacionamento entre os táxons do grupo Ocellatocoris ${ }^{+}$do cladograma de consenso de Ochlerini após a pesagem sucessiva $\left(\begin{array}{l}= \\ =\end{array}\right.$ sinapomorfia; $\mathbf{O}=$ homoplasia).

O grupo Herrichella* apresentou melhora na definição de seus grupos internos após a pesagem sucessiva e dois outros grupos monofiléticos são reconhecidos (Fig. 4): Alathetus + A. parvus, suportado por cinco homoplasias, presença de processos anteoculares $\left(4_{1}\right)$, comprimento do primeiro artículo antenal igual ao dobro da sua largura $\left(18_{2}\right)$, perda das expansões lobulares do pigóforo $\left(35_{0}\right)$, presença de projeção cônica mediana no bordo ventral do pigóforo
(36, ) e vésica helicoidal (61 1 ); e Barola*, suportado por três homoplasias, ocelos muito próximos aos olhos $\left(7_{1}\right)$, perda das expansões lobulares do pigóforo $\left(35_{0}\right)$ e membrana posterior ao urotergito VII do macho expandida em direção posterior $\left(40_{1}\right)$.

Independentemente do critério de análise adotado, com ou sem pesagem sucessiva, quatro grupos monofiléticos podem ser reconhecidos em Pseudadoxoplatys $^{+}$(Figs. 1-4): Eritrachys + Phereclus, 
Herrichella*, Schaefferella* e Moncus+O. handlirschi. Schaefferella* compartilha jugas contíguas no ápice $\left(3_{1}\right)$, homoplástico com Discocephala e com Stictochilus; processos anteoculares agudos $\left(4_{1}\right)$, homoplástico com Alathetus + A. parvus; presença de projeção pós-anal ventral no segmento $\mathrm{X}$ dos machos $\left(38_{1}\right)$, homoplástico com Parochlerus; ductus receptaculi anterior à área vesicular de mesmo calibre que a parede intermediária dessa área (49,), homoplástico com Alitocoris "a"; pars intermedialis e capsula seminalis equivalendo a aproximadamente a metade do comprimento da área vesicular (52, ), homoplástico com Adoxoplatys,
Alitocoris "a" e Parochlerus; vésica helicoidal (61 $)$, homoplástico com Alathetus+A. parvus e com reversão em Uvaldus; e uma reversão, terço mediano longitudinal ventral do abdome convexo $\left(32_{0}\right)$.

Schaefferella* contém quatro grupos monofiléticos (Fig. 4): Uvaldus ${ }^{+}$, suportado pela projeção mediana cônica no bordo ventral do pigóforo $\left(36_{1}\right)$, homoplástico com Alathetus + A. parvus; ductus receptaculi estreitado na área vesicular, em direção à pars intermedialis $\left(51_{1}\right)$, homoplástico com A. parvus; e vésica envolvida por conjuntiva membranosa $\left(62_{1}\right) ;$ Brachelytron $^{+}$, que compartilha uma sinapomorfia, comprimento do primeiro

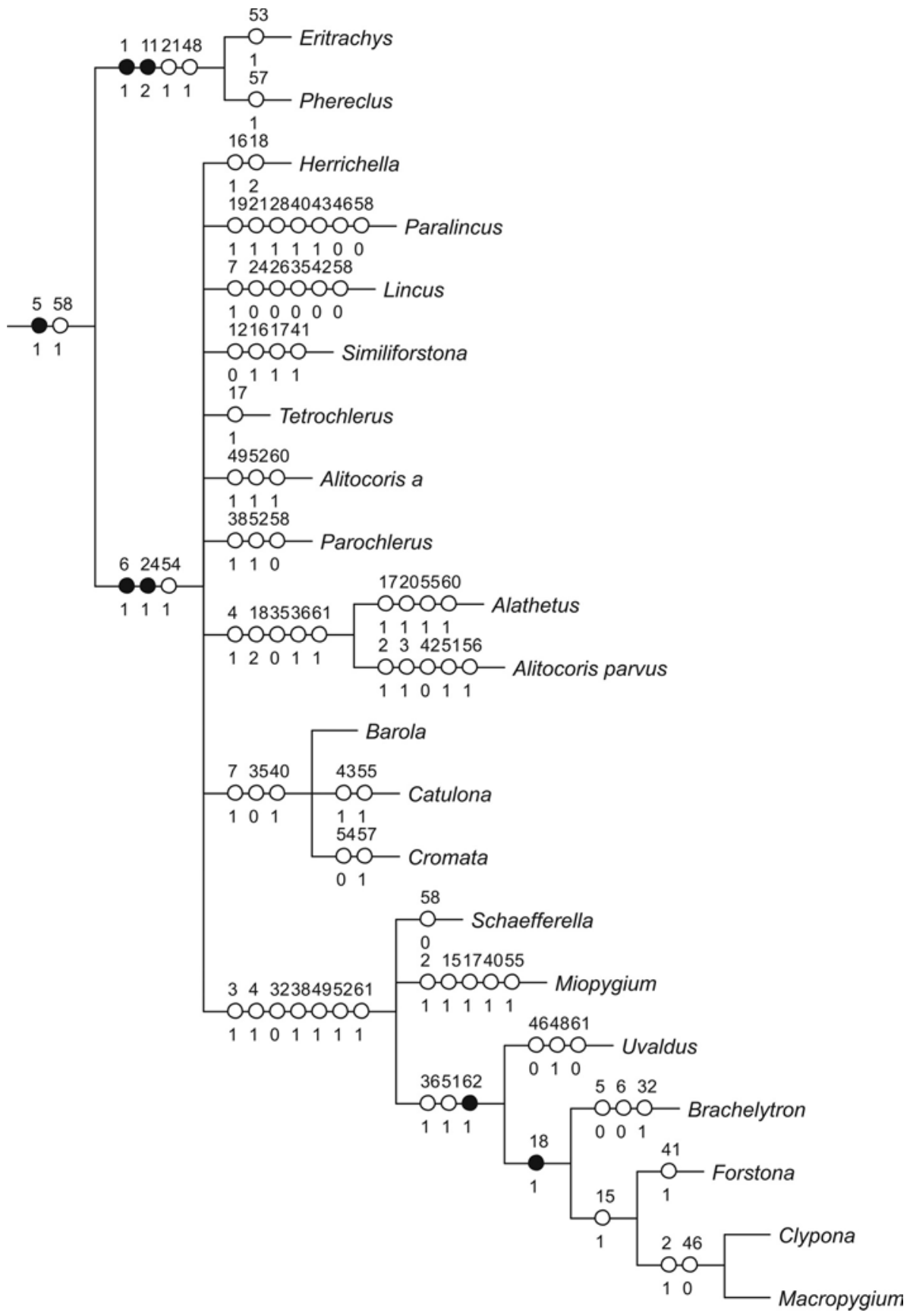

Fig. 4. Relacionamento entre os táxons do clado reunindo Eritrachys+Phereclus e Herrichella* do cladograma de consenso de Ochlerini após a pesagem sucessiva ( $=$ sinapomorfia; $\mathbf{O}=$ homoplasia). 


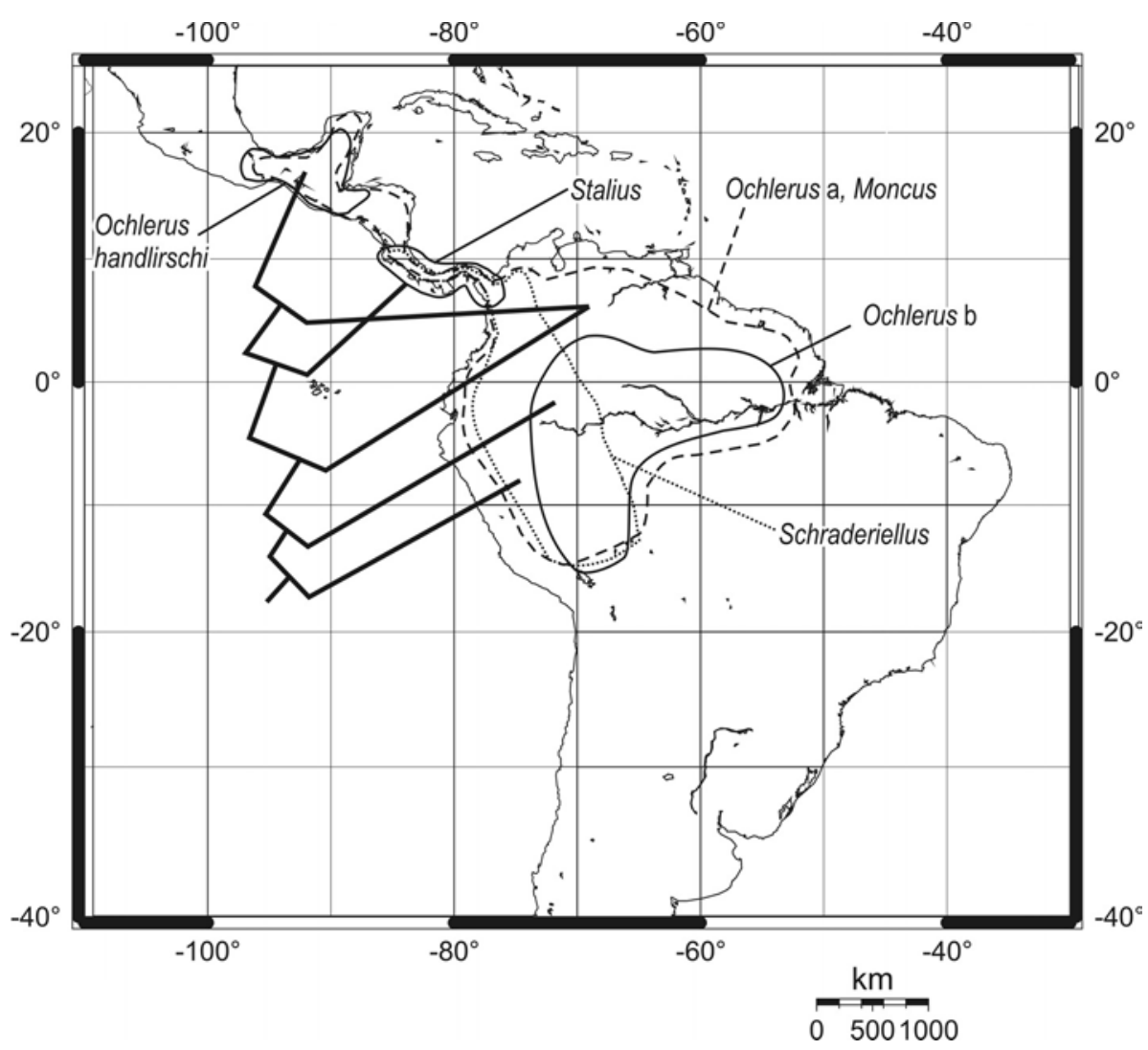

Fig. 5. Distribuição geográfica do grupo Schraderiellus ${ }^{+}$.

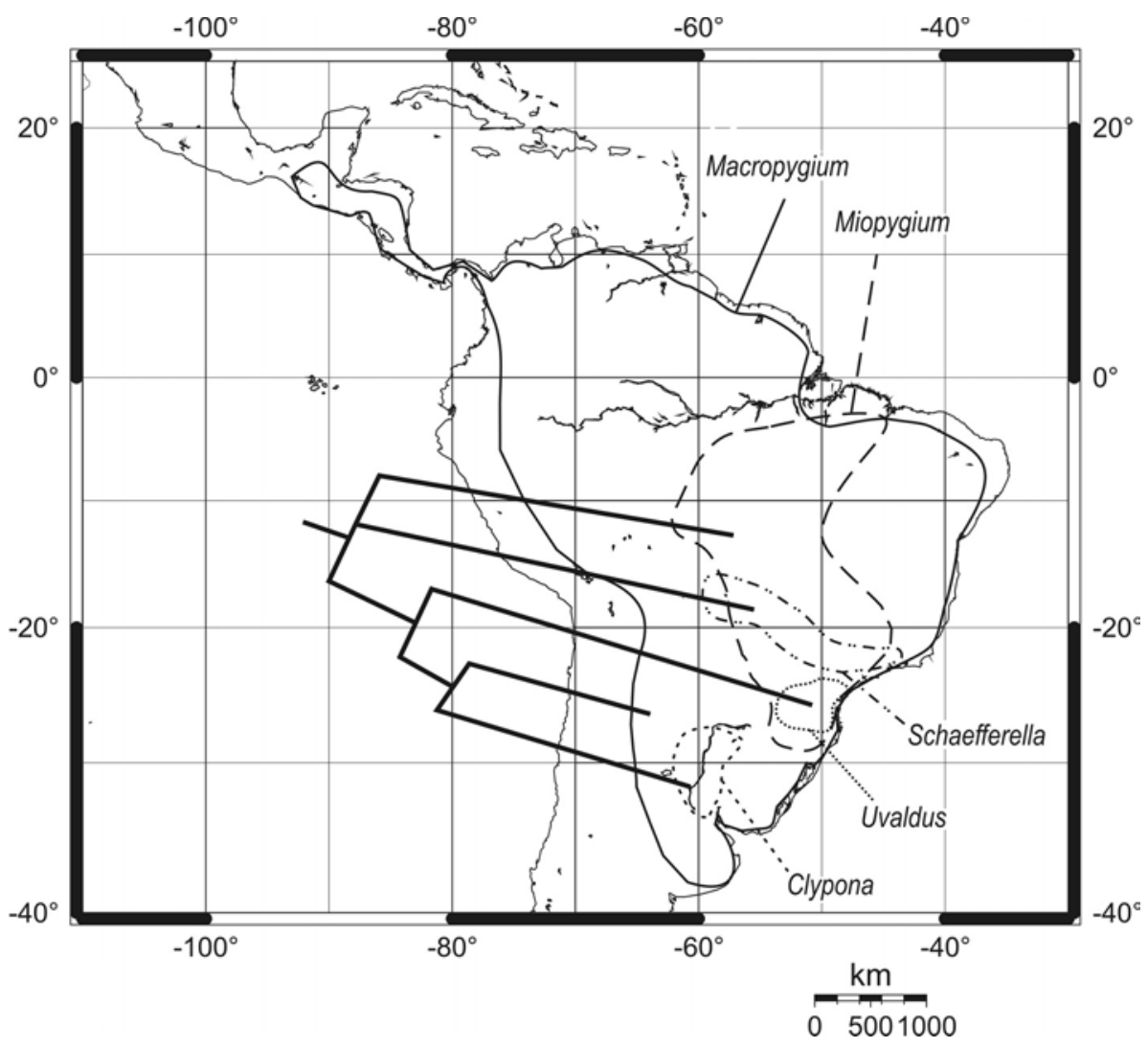

Fig. 6. Distribuição geográfica do grupo Schaefferella*. 

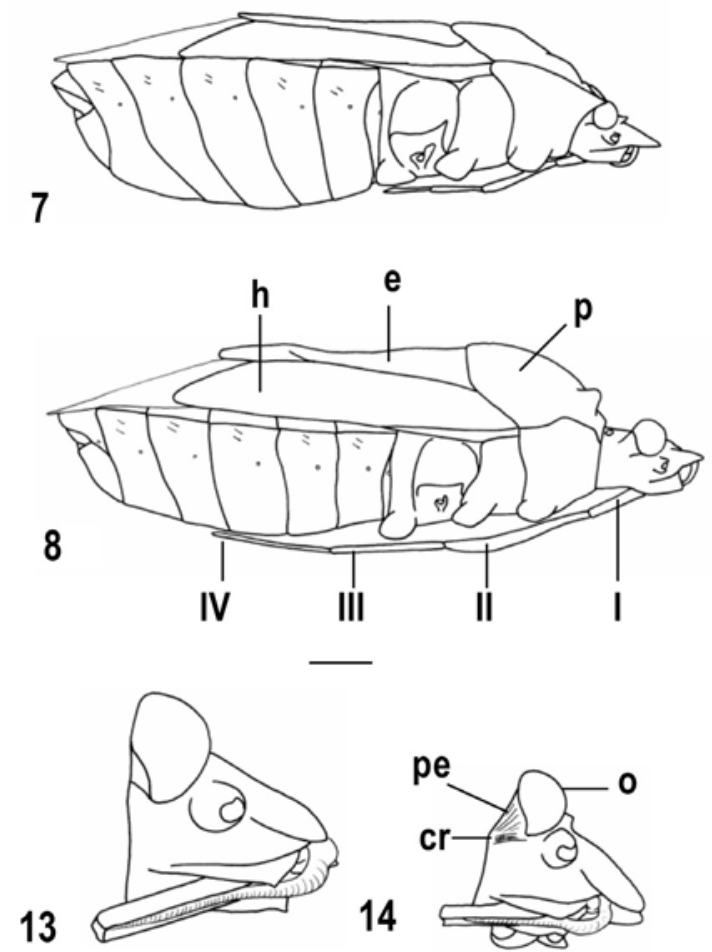

14

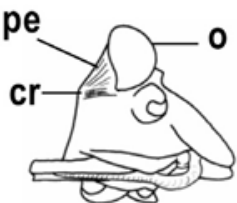

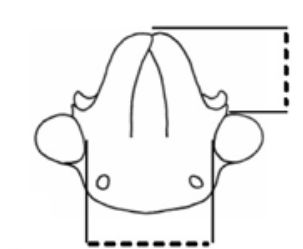

dio
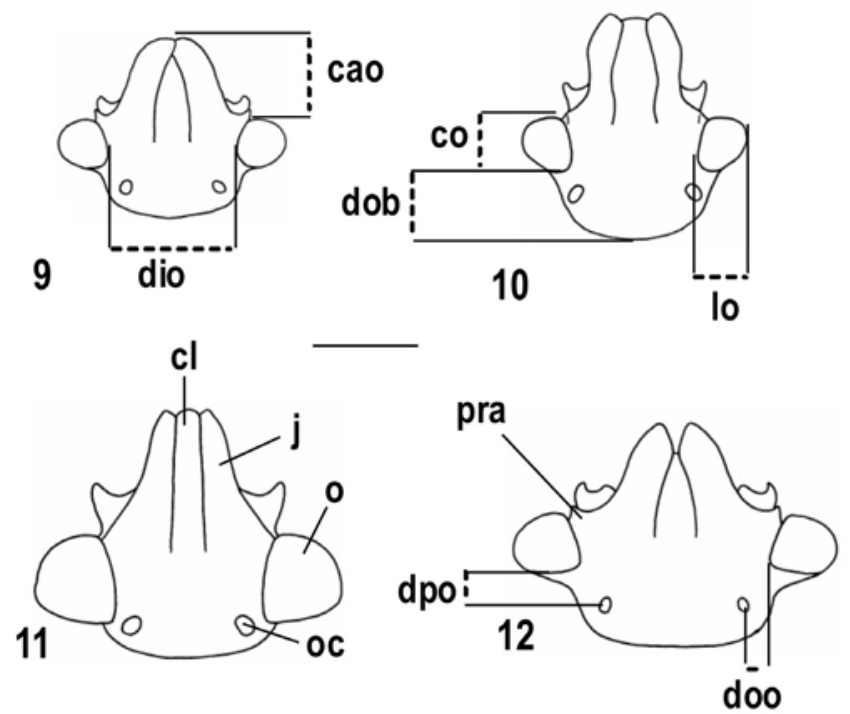

doo

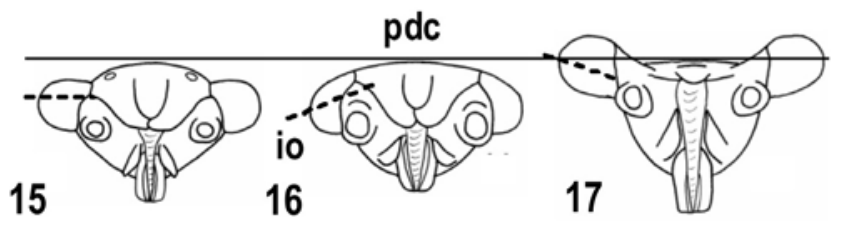

Figs. 7-17. Estruturas morfológicas representando os estados dos caracteres 1 a 11,15 e 16 . Figs. 7-8, corpo em perfil, pernas e antenas removidas: 7, Macropygium reticulare; 8, Eritrachys bituberculata (I, II, III, IV, primeiro a quarto artículos rostrais; e, escutelo, h, hemiélitro; p, pronoto); figs. 9-17, cabeça, 9-12, vista dorsal; 13-14, vista ventro-lateral; 15-17, vista frontal; 9, 15, Uvaldus concolor; 10, Catulona pensa; 11, 13, Ochlerus cinctus; 12, Miopygium ciclopeltoides; 14, Schaefferella incisa; 16, Adoxoplatys comis; 17, Eritrachys bituberculata (cao, comprimento anteocular; cl, clípeo; co, comprimento do olho; cr, carena; dio, distância interocular; dob, distância olho-base da cabeça; doo, distância lateral olho-ocelo; dpo, distância longitudinal olho-ocelo; io, inclinação do olho; j, jugas; lo, largura do olho; o, olho; oc, ocelo; pdc, plano dorsal da cabeça; pe, pedúnculo; pra, processo anteocular). Escalas = 0,6 mm.
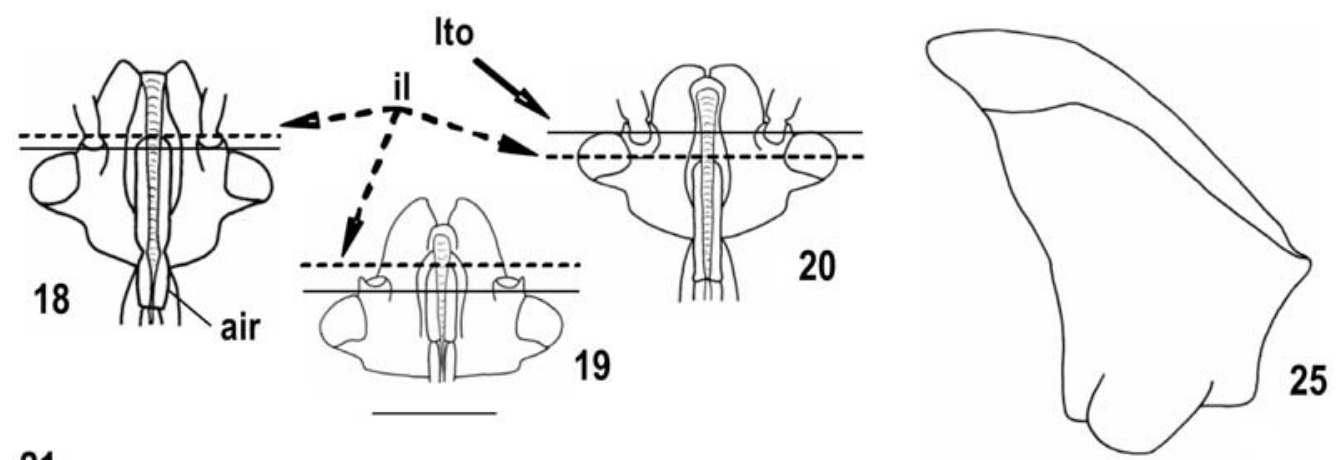

21
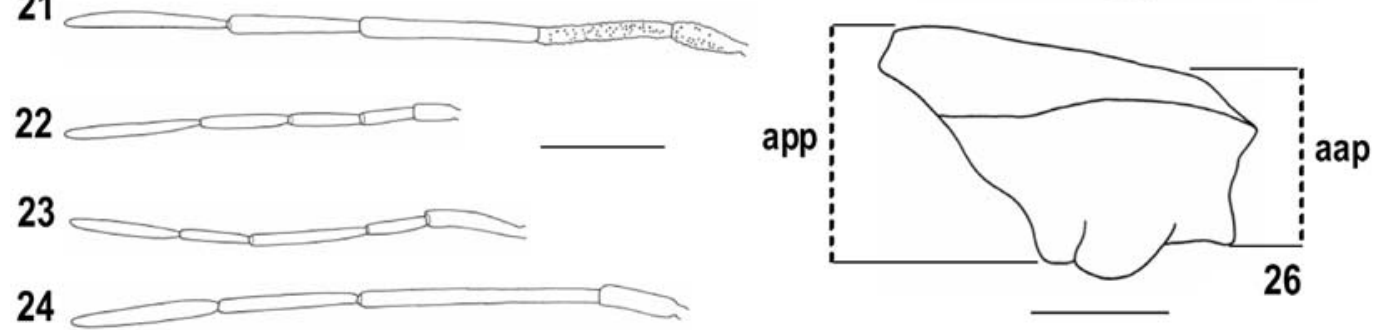

Figs. 18-26. Estruturas morfológicas representando os estados dos caracteres 12 a 14 e 17 a 20 . Figs. 18-20, cabeça, vista ventral: 18 , Neoadoxoplatys saileri; 19, Janeirona stali; 20, Schaefferella incisa (air, artículo intercalar do rostro; il, inserção do lábio; lto, linha tangente à margem anterior dos olhos); figs. 21-24, antena esquerda, vista dorsal: 21, Adoxoplatys willineri; 22, Macropygium reticulare; 23, Eritrachys bituberculata; 24, Alathetus haitiensis; figs. 25-26, protórax, vista lateral direita: 25, Schaefferella incisa; 26, Adoxoplatys willineri (aap, altura anterior do pronoro; app, altura posterior do pronoto). Escalas $=0,6 \mathrm{~mm}$. 
artículo antenal equivalente ao dobro da sua largura $\left(18_{1}\right)$; Forstona $^{+}$, suportado pelo segundo artículo rostral curto (15 $)$, homoplástico com Miopygium Breddin, 1904 e visto em parte de Halyini e de Discocephalini, além de vários gêneros de Pentatomidae (p. ex., Edessa Fabricius, 1803; Brachystethus Laporte, 1832; Paratibraca Campos \& Grazia, 1995; Glyphepomis Berg, 1891); o grupo apical, Clypona + Macropygium, compartilha o acentuado afastamento interocular em relação ao comprimento da cabeça (2 $2_{1}$, homoplástico com Miopygium e A. parvum e visto em diversos gêneros de Discocephalini reconhecíveis pela cabeça ampla, "broadheaded discocephalines" segundo Rolston (1990), e uma reversão, segmento $X$ da fêmea exposto $\left(46_{0}\right)$.

A análise dos grupos formados no cladograma evidencia o merofiletismo dos gêneros Ochlerus (polifilético), Stalius e Alitocoris (parafiléticos), e a necessidade de revisão e análise cladística desses gêneros.

A comparação entre os grupos formados no cladograma com as aproximações empíricas feitas por outros autores para os gêneros da tribo permite algumas considerações: o parentesco entre Adoxoplatys e Neoadoxoplatys (Kormilev, 1956; Ruckes, 1958; Rolston, 1992) é suportado; o grupo proposto por
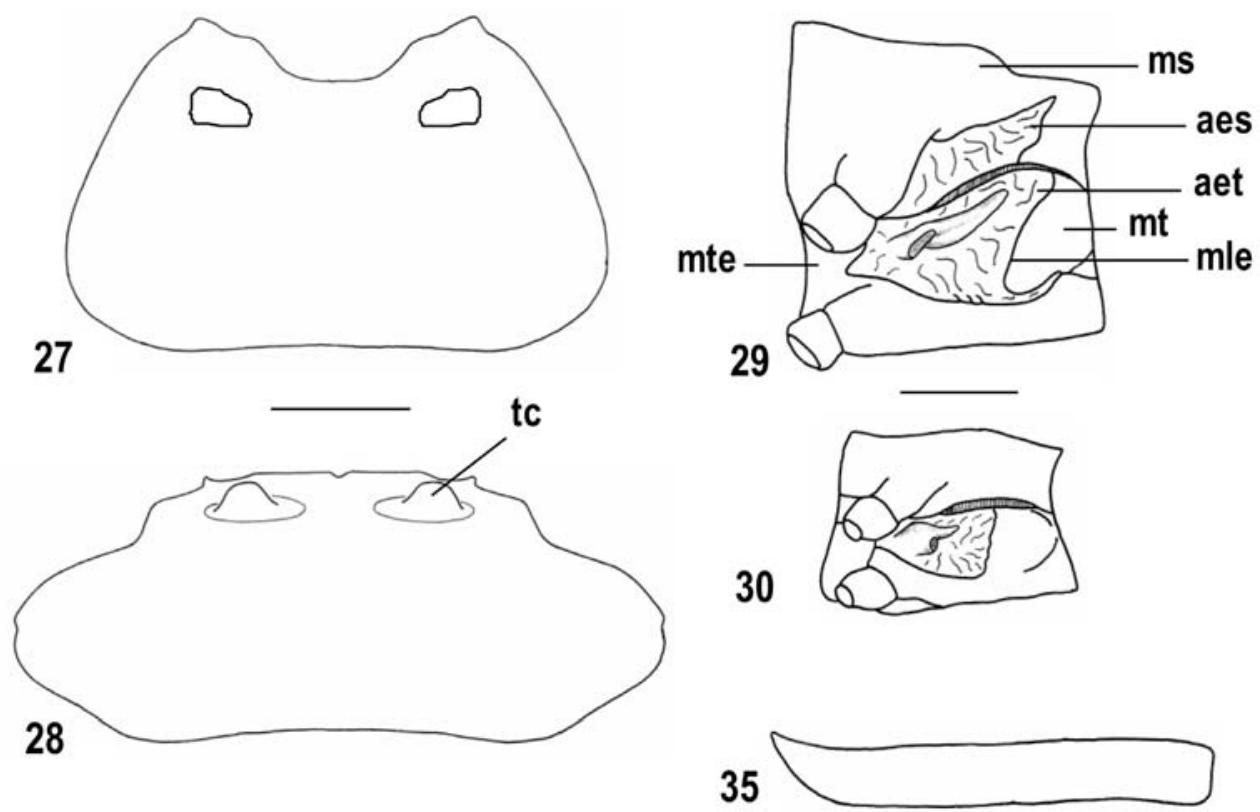

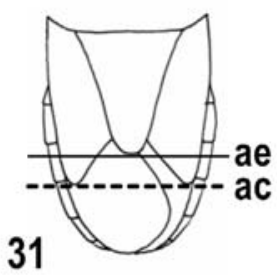

32

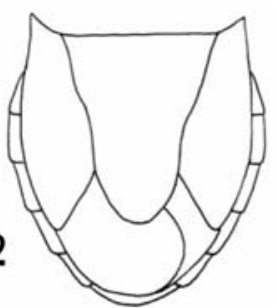

33

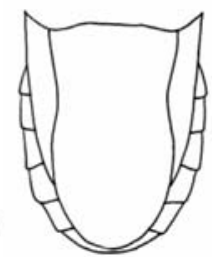

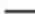

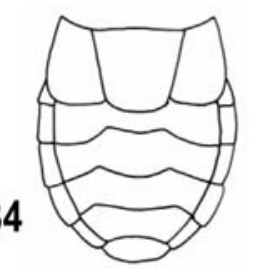

36

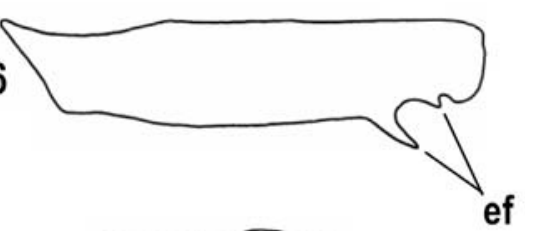

37

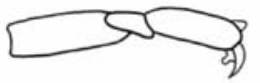

38

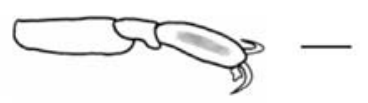

39

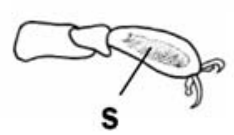

Figs. 27-39. Estruturas morfológicas representando os estados dos caracteres 21 a 24 e 27 a 30. Figs. 27-28, pronoto, vista dorsal: 27, Adoxoplatys willineri; 28, Eritrachys bituberculata (tc, tubérculos das cicatrizes); figs. 29-30, mesopleura e metapleura direitas, vista ventro-lateral: 29, Janeirona stali; 30, Cromata ornata (aes, área evaporatória mesopleural; aet, área evaporatória metapleural; mle, margem lateral da área evaporatória; ms, mesopleura; mt, metapleura; mte, metasterno); figs. 31-34, escutelo, hemiélitro e abdome, esquemáticos, vista dorsal: 31, Adoxoplatys comis; 32, Ochlerus cinctus; 33, Moncus obscurus; 34, Brachelytron angelicus (ac, ápice do cório; ae, ápice do escutelo); figs. 35-36, profêmur direito, vista lateral: 35, Moncus obscurus; 36, Neoadoxoplatys saileri (ef, espinhos femorais); figs. 37-39, fêmea, metatarso direito, vista dorso-lateral: 37, Janeirona stali; 38, Neoadoxoplatys saileri; 39, Catulona pensa (s, sulco tarsal). Escalas $=3 \mathrm{~mm}$. 

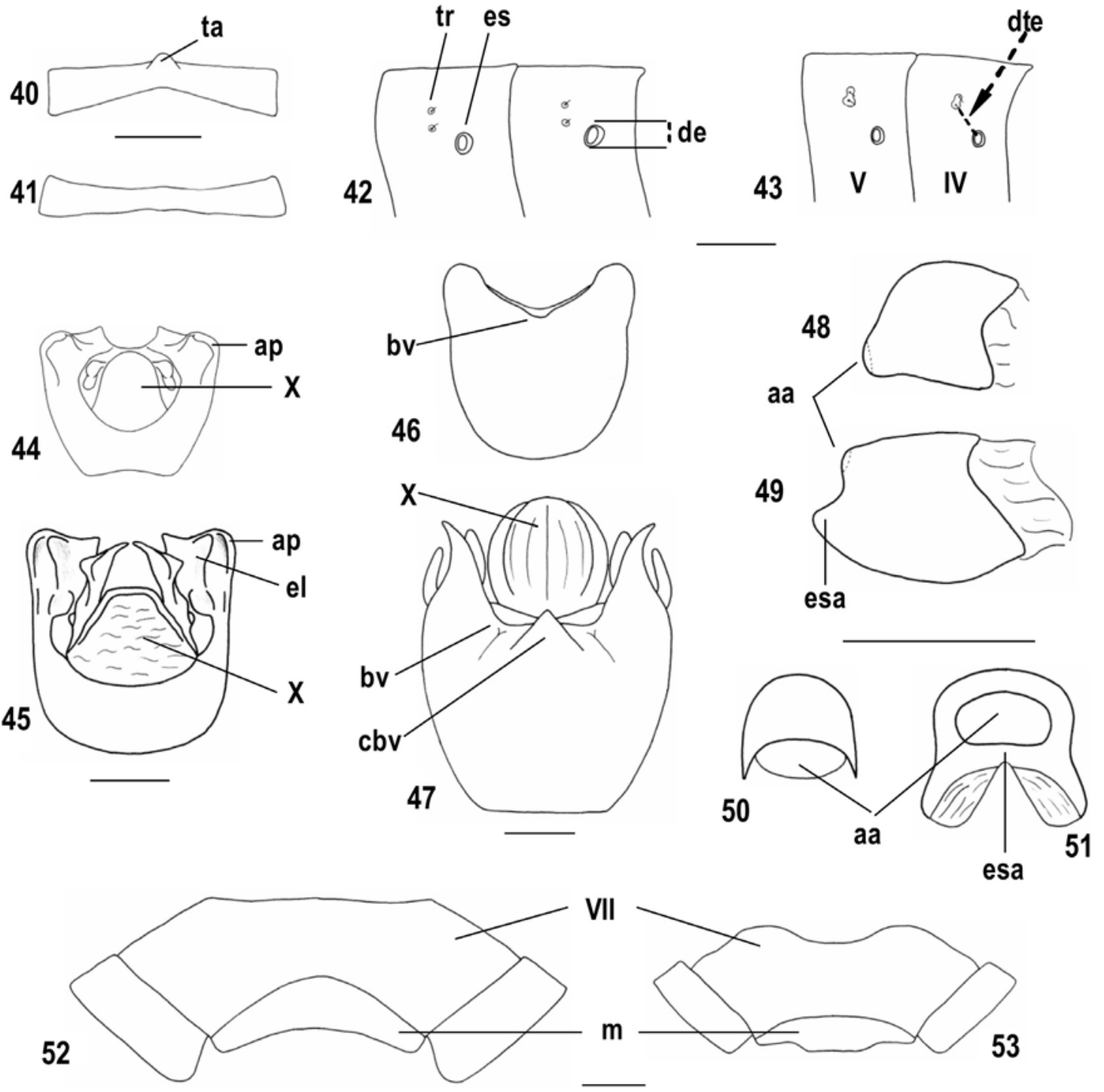

Figs. 40-53. Estruturas morfológicas representando os estados dos caracteres 31, 33 e 35 a 40. Figs. 40-41, terceiro urosternito, vista ventral: 40, Adoxoplatys comis; 41, Cromata ornata (ta, tubérculo abdominal); figs. 42-43, quarto e quinto urosternitos, vista ventrolateral: 42, Janeirona stali; 43, Schaefferella incisa (IV, quarto urosternito; V, quinto urosternito; de, diâmetro do espiráculo; dte, distância tricobótrio-espiráculo; es, espiráculo; tr, tricobótrios); figs. 44-47, pigóforo, 44-45; vista dorsal, 46-47, vista ventral: 44, Stictochilus tripunctatus; 45, Moncus obscurus; 46, Catulona pensa; 47, Macropygium reticulare (X, segmento X; ap, ângulo pósterolateral; bv, bordo ventral; cbv, projeção cônica mediana do bordo ventral; el, expansão lobular); figs. 48-51, macho, segmento X, 48-49, vista lateral; 50-51, vista posterior: 48, 50, Neoadoxoplatys saileri; 49, 51, Uvaldus concolor (aa, abertura anal; esa, espessamento subanal); figs. 52-53, macho, sétimo urotergito: 52, Janeirona stali, 53, Miopygium cyclopeltoides (VII, sétimo urotergito; m, membrana). Escalas $=0,6 \mathrm{~mm}$ (figs. 40-43), 0,2 mm (figs. 44-53).

Rolston (1992), contendo Ochlerus, Moncus Stål, 1867, Orbatina, Schraderiellus e Stalius, é suportado com a inclusão de Coranda e tendo Ocellatocoris como grupoirmão; dois outros grupos propostos por RoLSTON (1992), o primeiro composto por Clypona, Miopygium, Forstona e Uvaldus, o segundo por Schaefferella Spinola, 1850, Alitocoris e Macropygium, são isoladamente grupos merofiléticos, mas juntos formam um grupo monofilético com a inclusão de Brachelytron e a exclusão de Alitocoris. A proposição de BREDDIN (1904), de que Parochlerus e Tetrochlerus seriam gêneros afins e semelhantes a Ochlerus, não é sustentada. Rolston (1992) observou que aqueles gêneros provavelmente não eram próximos a Ochlerus, mas que seriam suficientemente próximos entre si a ponto de "formar um grupo à parte dentro de Ochlerini”, afirmação que não é suportada por nossas análises. De fato, os dois gêneros em questão têm apenas uma aparência geral semelhante, o que provavelmente levou os autores a considerá-los próximos. Assim, o resultado obtido apresenta certa congruência com grupos propostos anteriormente, em especial Orbatina ${ }^{+}$, Schaefferella ${ }^{+}$e Adoxoplatys + Neoadoxoplatys. As politomias observadas eram até certo ponto esperadas, dado o alto número de táxons com caracteres não- 

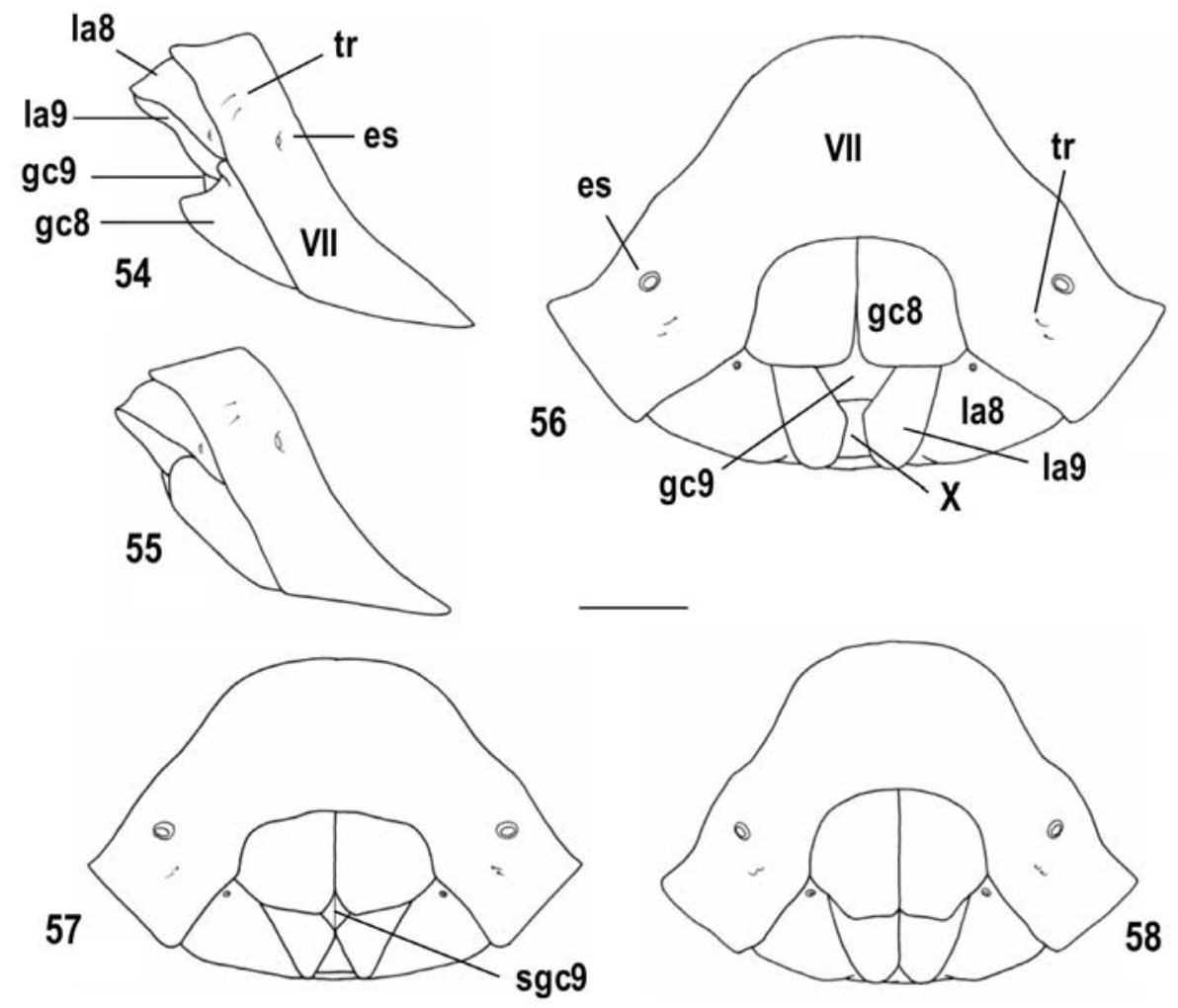

Figs. 54-58. Estruturas morfológicas representando os estados dos caracteres 41 a 46. Fêmea, placas genitais, 54-55, vista lateral; 56-58, vista ventral: 54, Similiforstona bella; 55, Macropygium reticulare; 56, Janeirona stali; 57, Cromata ornata; 58, Catulona pensa (VII, sétimo urosternito; gc8, gonocoxitos 8; gc9, gonocoxitos 9; la8, laterotergitos 8, la9; laterotergitos 9; es, espiráculo; sgc9, sutura dos gonocoxitos 9; tr, tricobótrio). Escalas $=1 \mathrm{~mm}$.

observados, destacando que os caracteres 35 a 63 referem-se a estruturas genitais externas e internas, tanto masculinas quanto femininas. Isso, além de reduzir a congruência dos dados, fez com que as distribuições dos estados dos caracteres sexuais sejam tentativas, sendo apenas dessa forma que as interpretações feitas neste trabalho devem ser consideradas. A correta distribuição desses caracteres, entretanto, poderá ser elucidada com a descoberta de machos e fêmeas das espécies de todos os gêneros incluídos neste estudo, o que permitirá um refinamento da análise.

Análise biogeográfica. A partir do cladograma de consenso obtido após a pesagem sucessiva e das áreas de distribuição de grupos monofiléticos de Ochlerini, foram construídos cladogramas biológicos de área (Figs. 5, 6).

Muitos dos táxons estudados apresentam simpatria, com maior ou menor superposição das áreas de ocorrência na América Central e América do Sul, dificultando a elucidação dos eventos vicariantes. As espécies do grupo Schraderiellus ${ }^{+}$(Fig. 5) distribuem-se do sul do México ao norte e noroeste da América do Sul, alcançando o norte da Bolívia, limitando-se ao norte pela Sierra Madre e ao sul pelas bacias dos rios Amazonas, Madeira e Mamoré. Segundo a divisão da Região Neotropical proposta por Morrone (2004), essa distribuição corresponde aos domínios Mesoamericano e Noroeste da América do Sul da sub-região Caribenha e à sub-região Amazônica. As espécies do gênero basal,
Schraderiellus, ocorrem do sul do domíno Mesoamericano (província Leste da América Central) ao oeste da sub-região Amazônica, limitadas às encostas orientais dos Andes, apresentando alguma simpatria com as espécies de Ochlerus "b" no oeste das províncias de Imeri, Madeira, Várzea e Pantanal. As espécies desse último grupo se estendem mais a leste, ao longo das províncias de Imeri, Várzea, Roraima e Amapá e não alcançam, a oeste, as encostas orientais do Andes. As espécies de Ochlerus "a" e de Moncus apresentam ampla distribuição nas duas sub-regiões, sendo simpátricas com as espécies dos grupos anteriores e presentes, também, no norte de cada uma das duas sub-regiões. Dois gêneros apresentam distribuição mais restrita: as espécies de Stalius ocorrem apenas na província Leste Americana, do sul da Guatemala ao Panamá, enquanto que Ochlerus handlirschi ocorre no norte do domínio Mesoamericano, nas províncias do Golfo do México, Península de Yucatán e Chiapas. A distribuição dos táxons de Schraderiellus ${ }^{+}$ pode ser o resultado de sucessivas dispersões a partir da sub-região Amazônica. Seus limites ao sul, correspondendo às bacias dos rios Amazonas, Madeira e Mamoré, podem ser explicados pela possível origem e evolução independentes das biotas do noroeste e do sudeste da Amazônia propostas por AmOrim (2001), estando as espécies deste clado associadas às primeiras.

As espécies do grupo Schaefferella* (Fig. 6) distribuem-se do sul do México ao nordeste da Argentina, limitando-se ao norte pela Sierra Madre, a oeste pelos 


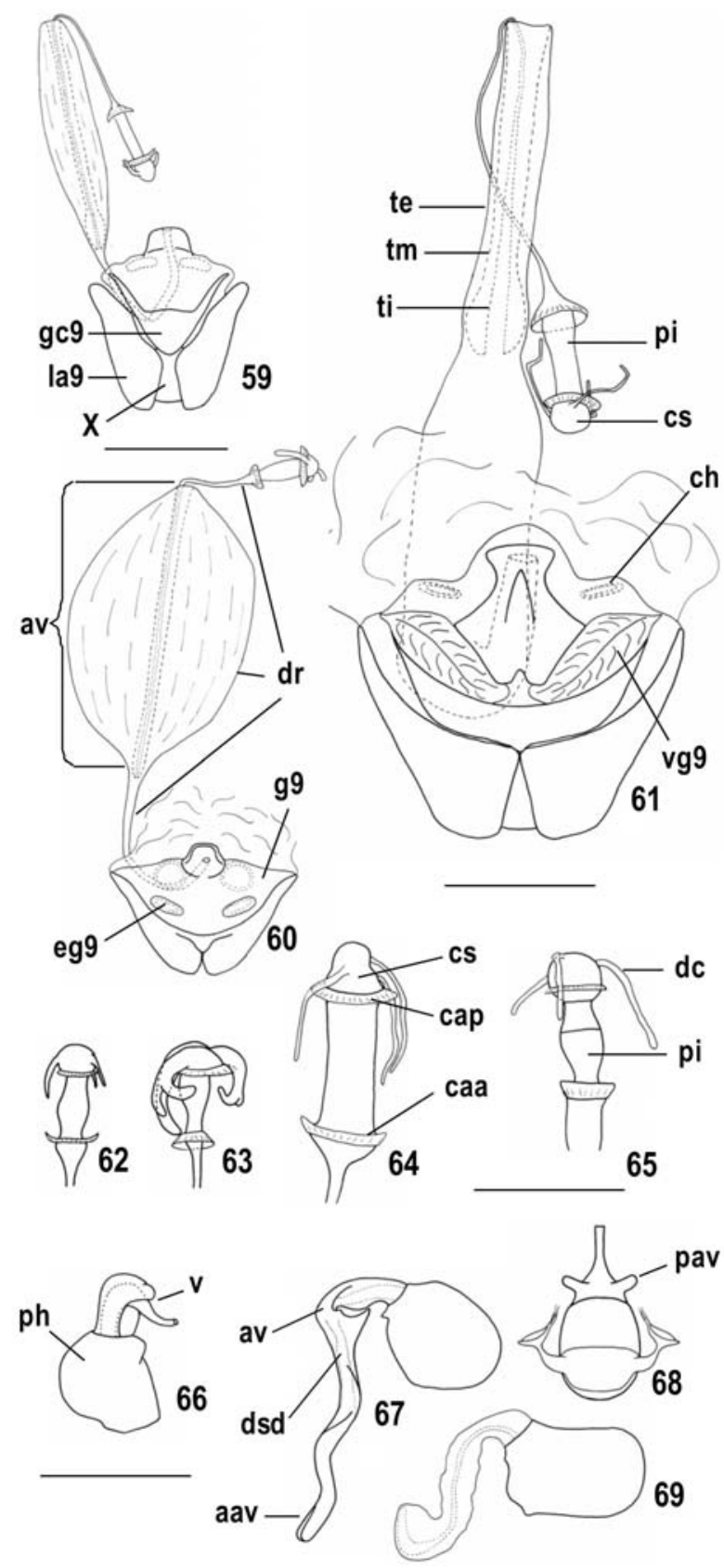

Figs. 59-69. Estruturas morfológicas representando os estados dos caracteres 47 a 63. Figs. 59-61, fêmea, gonocoxitos 9, laterotergitos 9, gonapófises 9 e vias genitais ectodérmicas: 59, Adoxoplatys willineri; 60, Stalius tartareus; 61, Macropygium reticulare (X, segmento X; ch, chitinellipsen; cs, capsula seminalis; dr, ductus receptaculi; te, tubo externo da área vesicular; g9, gonapófise 9 ; gc9, gonocoxitos 9; ti, tubo interno da área vesicular; la9, laterotergitos 9; tm, tubo médio da área vesicular; pi, pars intermedialis; vg9, microestruturas coniformes da gonapófise 9 eg9, espessamento da gonapófise 9; av, área vesicular); figs 62-65, fêmea, ápice do ductus receptaculi: 62, Ochlerus cinctus; 63, Phereclus pluto; 64, Miopygium cyclopeltoides; 65, Ochlerus handlirschi (caa, crista anelar anterior; cs, capsula seminalis; cap, crista anelar posterior; pi, pars intermedialis; dc, dentes da capsula seminalis); figs. 66-69, macho, phallus, respectivamente vistas dorso-lateral, lateral, dorsal e lateral: 66, Neoadoxoplatys saileri; 67, Miopygium cyclopeltoides; 68, Stalius tartareus; 69, Uvaldus concolor (aav, abertura apical da vésica; av, articulação da vésica; dsd, ductus seminis distalis; ph, phallotheca; v, vésica; pav, projeções laterais da vésica). Escalas $=2 \mathrm{~mm}$.
Andes e ao sul pelo limite do pampa sulino. Tal distribuição corresponde aos domínios Mesoamericano e Noroeste da América do Sul da sub-região Caribenha, e às sub-regiões Amazônica, Chaquenha e Paranaense. Entretanto, essa ampla distribuição resulta de apenas um táxon, Macropygium, o qual está em simpatria com os demais táxons do grupo, esses apresentando áreas de ocorrência mais restritas. Schaefferella ocorre em simpatria com Miopygium no leste da província do Pantanal, sul da província do Cerrado e centro da província da Mata Paranaense, áreas de cerrado com a presença de matas de galeria, alcançando a leste a província da Mata Atlântica Brasileira. As espécies de Miopygium estendem-se mais ao norte ao longo das províncias do Cerrado e do Pantanal até o sul da bacia amazônica, nas províncias de Madeira, Tapajós-Xingu e Pará; e mais ao sul até o norte do Rio Grande do Sul, ao longo das províncias da Mata Paranaense e da Mata de Araucaria angustifolia. Nessas áreas, Uvaldus ocorre em simpatria com Miopygium, sendo um táxon restrito à sub-região Paranaense, distribuído no sul da província da Mata Paranaense e no centro-norte da província da Mata de Araucaria angustifolia, alcançando a província da Mata Atlântica Brasileira. Clypona é restrito à subregião Chaquenha, sendo conhecido do nordeste da Argentina, províncias do Chaco e do Pampa. Cabe destacar que os gêneros monotípicos Forstona e Brachelytron são conhecidos apenas dos holótipos, sendo seus registros restritos às localidades-tipo, respectivamente Vilhena (Rondônia) e Corupá (Santa Catarina). A distribuição dos táxons de Schaefferella* pode estar relacionada com uma origem em biotas na subregião Paranaense, com posterior expansão ao norte em direção ao sudeste da sub-região Amazônica e ao sul em direção à sub-região Chaquenha. Nesse caso, as distribuições restritas de Uvaldus e Clypona poderiam ter resultado de eventos vicariantes que separaram as sub-regiões Paranaense e Chaquenha. A ampla distribuição de Macropygium poderia ser conseqüência da invasão de novas áreas partindo do sul da América do Sul.

A ampliação do conhecimento acerca da distribuição geográfica de táxons pouco representados em coleções, bem como estudos sobre a biogeografia dos táxons com ampla distribuição, como Lincus e Ochlerus, poderão trazer novas contribuições para o conhecimento dos padrões biogeográficos na região Neotropical.

Agradecimentos. Aos dois consultores anônimos, pelas inestimáveis contribuições. Aos Drs. Sônia Aparecida Casari (USP), Sergio Antonio Vanin (USP), Dalton de Souza Amorim (USP) e Pedro Gnaspini Netto (USP), pelas valiosas críticas e sugestões no trabalho original. A todos os colegas e curadores de coleções que gentilmente permitiram o empréstimo do material. À FAPESP (processo $\mathrm{n}^{\circ}$ 96/1328-2) e ao $\mathrm{CNPq}$, pelo apoio financeiro.

\section{REFERÊNCIAS BIBLIOGRÁFICAS}

Ahmad, I. \& Khan, A. N. 1983. A revision of the genus Stenozygum Fieber (Pentatomidae, Strachini) from the Oriental and Australian regions, with reference to zoogeography and phylogeny. Australian Journal of Zoology 31:581-605. Amorim, D. S. A. 1982. Classificação por seqüenciação: uma 
proposta para a denominação dos ramos retardados. Revista Brasileira de Zoologia 1(1):1-9.

1994. Group*: an additional artifact for phylogenetic sequenced classifications. Revista Nordestina de Biologia 8(1):35-38. 2001. Dos Amazonias. In: Llorente-Bousquets J.; Morrone J.

J. \& Flores, O. eds. La biogeografia en America Latina. Teorías, conceptos, métodos y aplicaciones. México, Facultad de Ciencias, UNAM. p.245-255.

Arnett, R. H.; Samuelson, G. A. \& Nishida, G. M. 1993. The insect and spider collections of the World. Gainesville, Saint Lucie. 316p.

Barcellos, A. \& Grazia, J. 2003. Cladistic analysis and biogeography of Brachystethus Laporte (Heteroptera, Pentatomidae, Edessinae). Zootaxa 256:1-14.

BredDin, G. 1904. Neue Rhynchoteneusbeute aus Süd-Amerika. Societas Entomologica 18(20):153-154

Campos, L. A. 2005. A new species of Cromata Rolston from Brazilian and Venezuelan Amazon Forest (Hemiptera: Pentatomidae: Discocephalinae). Zootaxa 820:1-6.

Campos, L. A. \& Grazia, J. 2000. Descrição de um novo gênero de Ochlerini Rolston (Heteroptera, Pentatomidae, Discocephalinae). Revista Brasileira de Zoologia 17(3):789-793.

2001. Um novo gênero de Ochlerini do sul do Brazil (Heteroptera, Pentatomidae, Discocephalinae). Iheringia, Série Zoologia, 90:55-58

Campos, L. A.; Grazia, J. \& Greve, C. 2004. Notes on Catulona Rolston, 1992, and the description of a new species from Brazil (Hemiptera, Pentatomidae, Discocephalinae). Zootaxa 404:1-7.

CARpenter, J. M. 1988. Choosing among multiple equally parsimonious cladograms. Cladistics 4:291-296.

1994. Successive weighting, reliability and evidence. Cladistics 10:215-220.

Couturier, G. \& KAHN, F. 1989. Bugs of Lincus spp. vectors of Marchitez and Hartrot (oil palm and coconut diseases) on Astrocaryum spp., Amazonian native palms. Principes 33(1): 19-20.

Desmier-DE-Chenon, R. 1984. Recherches sur le genre Lincus Stål, Hemiptera Pentatomidae Discocephalinae, et son rôle éventuel dans la transmission de la Marchitez du palmier à huile et du Hart-Rot du cocotier. Oléagineux 39(1):1-6.

Dollet, M.; Alvanil, F.; Diaz, A.; Louvet, C.; Gargani, D.; Segur, C.; Muller, E.; Julia, J. F.; Calvache, H.; Renard, J. L. \& Maldes, J. M 1993. Les Pentatomides Vecteurs des Trypanosomes Associes au Hartrot du Cocotier et Marchitez du Palmier. In: ANPP ed. Conférence Internationale sur les Ravageurs en Agriculture, $3^{\text {ème, }}$ Montpellier, Annales... Tome III, p. 1321-1328.

DupuIs, C. 1955. Les génitalia des Hémiptères-Héteroptères (Génitalia externes des deux sexes, voies ectodermiques femelles). Mémoirs du Museum National d'Histoire Naturelle (France), Nouvelle Série, Série A, Zoologie 6(4):183-278.

. 1970. Heteroptera. In: TuXEN, S. L. ed. Taxonomist's glossary of genitalia of insects. Copenhagen, Munksgaard. p.190-208.

FARRIS, J. S. 1969. A successive weighting approximations approach to character weighting. Systematic Zoology 18:374-385.

Fieber, F. X. 1860. Die europäischen Hemiptera. Halbflüger (Rinchota Heteroptera). Nach der analytischen Methode bearbeitet. Wien, Gerold. 444p.

Fortes, N. D. F. DE \& Grazia, J. 2005. Revisão e análise cladística de Serdia Stål (Heteroptera, Pentatomidae, Pentatomini). Revista Brasileira de Entomologia 49(3):294-339.

GAPUD, V. P. 1991. A generic revision of the subfamily Asopinae, with consideration of its phylogenetic position in the family Pentatomidae and superfamily Pentatomoidea (Hemiptera Heteroptera). Philippines Entomology 8(3):865-961.

Grazia, J. 1997. Cladistic analysis of the Evoplitus genus group of Pentatomini (Heteroptera: Pentatomidae). Journal of Comparative Biology 2(1):43-48.

Hasan, S. A. \& Kitching, I. A. 1993. A cladistic analysis of the tribes of the Pentatomidae (Heteroptera). Japanese Journal of Entomology 61(4):651-669.
Henry, T. 1997. Phylogenetic analysis of family groups within the infraorder Pentatomomorpha (Hemiptera: Heteroptera), with emphasis on the Lygaeoidea. Annals of the Entomological Society of America 90(3):275-301.

Humphries, C. J. \& Parenti, L. R. 1999. Cladistic Biogeography: interpreting patterns of plant and animal distributions. 2ed. Oxford, Clarendon. 199p.

Kormilev, N. A. 1956. Notas sobre Pentatomoidea neotropicales $\mathrm{V}$ (Hemiptera). Scientifica de los Institutos de Investigacion de San Miguel 4:3-7.

Llosa, J. F.; Couturier, G. \& Kahn, F. 1990. Notes on the ecology of Lincus spurcus and L. malevolus (Heteroptera: Pentatomidae: Discocephalinae) on Palmae in Forests of Peruvian Amazonia. Annales de la Société Entomologique de France (Nouvelle Série) 26(2):249-254.

Maddison, W. P.; Donoghue, M. J. \& Maddison, D. R. 1984. Outgroup analysis and parsimony. Systematic Zoology 33(1):83-103.

Morrone, J. J. 2004. Panbiogegrafía, componentes bióticos y zonas de transición. Revista Brasileira de Entomologia 48(2):149-162.

Morrone, J. J. \& CRISCI, J. V. 1995. Historical biogeography: introduction to methods. Annual Review of Ecology and Systematics 26:373-401.

Nixon, K. C. 2002. WinClada ver. 1.00.08. Ithaca, publicado pelo autor.

Ortega-Leon, G. \& Thomas, D. B. 2004. Two new species of Stalius Rolston (Heteroptera: Pentatomidae: Ochlerini) from Central America. Journal of the New York Entomological Society 112(2-3): 190-197.

PAge, R. D. M. 2001. NDE (NEXUS data editor for windows). Version 0.5.0 NDE- home page. Disponível em: <http:// taxonomy.zoology.gla.ac.uk/rod/NDE/nde.html>. Acesso em:12.2001.

Rolston, L. H. 1981. Ochlerini, a new tribe in Discocephalinae (Hemiptera: Pentatomidae). Journal of the New York Entomological Society 89(1):40-42.

1990. Key and diagnoses for the genera of 'broadheaded' discocephalines (Hemiptera: Pentatomidae). Journal of the New York Entomological Society 98(1):14-31.

1992. Key and diagnoses for the genera of Ochlerini (Hemiptera: Pentatomidae: Discocephalinae). Journal of the New York Entomological Society 100(1):1-41.

Rolston, L. H. \& McDonald, F. J. D. 1979. Keys and diagnoses for the families of Western hemisphere Pentatomoidea, subfamilies of Pentatomidae and tribes of Pentatominae (Hemiptera). Journal of the New York Entomological Society 87(3): 189-207.

Ruckes, H. 1958. New genera and species of neotropical discocephaline and halyine pentatomids (Heteroptera, Pentatomidae). American Museum Novitates 1868:1-27.

Schatfer, C. W. \& Ahmad, I. 1987. A cladistic analysis of the genera of the Lestonocorini (Hemiptera, Pentatomidae, Pentatominae). Proceedings of the Entomological Society of Washington 89(3):444-447.

Schun, R. T. \& Slater, J. A. 1995. True bugs of the World (Hemiptera: Heteroptera): classification and natural history. Ithaca, Cornell University. 336p.

STÅL, C. 1867. Bidrag till hemiptererans systematic. Öfversigt af Kongliga Vetenskaps-Akademiens Förhandlingar 24(7):491-560.

Swofford, D. L. 2003. PAUP*. Phylogenetic Analysis Using Parsimony (*and Other Methods). Version 4. Sunderland, Sinauer.

Thomas, D. B. 2000. Pentatomidae (Hemiptera). In: Llorente, J. E.; Gonzales, E. \& Papavero, N. eds. Biodiversidad, taxonomía y biogeografía de artrópodos de México: hacia una síntesis de su conocimiento. México, Universidad Autónoma de México. v. 2, p.335-352.

Watrous, L. E. \& Wheeler, Q. D. 1981. The outgroup comparison method of character analysis. Systematic Zoology 30(1): $1-11$ 